University of Nebraska - Lincoln

DigitalCommons@University of Nebraska - Lincoln

$7-1-2003$

\title{
Bulk and interfacial properties of a dipolar-quadrupolar fluid in a uniform electric field: A density-functional approach
}

V.B. Warshavsky

University of Nebraska-Lincoln

Xiao Cheng Zeng

University of Nebraska-Lincoln, xzeng1@unl.edu

Follow this and additional works at: https://digitalcommons.unl.edu/chemzeng

Part of the Chemistry Commons

Warshavsky, V.B. and Zeng, Xiao Cheng, "Bulk and interfacial properties of a dipolar-quadrupolar fluid in a uniform electric field: A density-functional approach" (2003). Xiao Cheng Zeng Publications. 34.

https://digitalcommons.unl.edu/chemzeng/34

This Article is brought to you for free and open access by the Published Research - Department of Chemistry at DigitalCommons@University of Nebraska - Lincoln. It has been accepted for inclusion in Xiao Cheng Zeng Publications by an authorized administrator of DigitalCommons@University of Nebraska - Lincoln. 


\title{
Bulk and interfacial properties of a dipolar-quadrupolar fluid in a uniform electric field: A density-functional approach
}

\author{
V. B. Warshavsky and X. C. Zeng \\ Department of Chemistry, University of Nebraska-Lincoln, Lincoln, Nebraska 68588, USA
}

(Received 17 February 2003; published 14 July 2003)

\begin{abstract}
We have studied the bulk and interfacial properties of a dipolar-quadrupolar fluid based on an extended modified mean-field density-functional theory. Effects of a uniform electric field on the bulk and interfacial properties are also studied. Results of the coexisting vapor-liquid densities, interfacial profiles of the density and orientation order parameters, the surface tension, and their dependence on the temperature, magnitude of molecule dipole and quadrupole moment, and the applied field are obtained. In general, we find that the applied field increases the critical temperature, broadens the vapor-liquid coexistence curves, and reduces the surface tension. We also find that if the quadrupole moment is positive, the reduction in the surface tension is greater when the applied field is in the direction from the vapor to the liquid phase than the reduction when the field is in the opposite direction. This apparent symmetry breaking by reversing the field direction may offer a molecular mechanism to explain the phenomenon of the sign preference in liquid droplet formation on charged condensation centers.
\end{abstract}

DOI: 10.1103/PhysRevE.68.011203

PACS number(s): 68.03. $-\mathrm{g}$, 82.60.Nh, 92.60.Jq, 92.60.Ls

\section{INTRODUCTION}

Many molecular fluids such as water, methanol, and hydrogen chloride possess both large molecular dipole and quadrupole moments. It is of both fundamental and practical importance to gain more knowledge of the bulk and interfacial properties of the molecular fluids as well as their dependence on the strength of the dipole and quadrupole moments, particularly when the fluids are subject to an external electric field. Theoretically, however, one can construct purely dipolar models such as the Lennard-Jones (LJ) plus a point dipole (known as the Stockmayer model), or purely quadrupolar models such as the the LJ plus a point quadrupole. With the purely dipolar or quadrupolar models, one can separately study the effects of dipole moment or quadrupole moment on the bulk and interfacial properties of the fluids. For example, a perturbation theory for both purely dipolar and quadrupolar fluids was developed by Haile et al. [1] to calculate the surface tension. More thorough reviews of the theoretical studies of purely dipolar fluids can be found in Refs. [2-4]. Bulk properties of dipolar fluids in an external field have been studied by many workers [5-9].

Several theoretical and computer-simulation studies of the properties of dipolar-quadrupolar fluids, including the free energy $[10,11]$, liquid structure [12], and phase equilibria [13], have been reported for model dipolar-quadrupolar fluid systems. Patey and Valleau [11] calculated the free energy and radial distribution function by using a Monte Carlo (MC) simulation method. Phase equilibria of the LJ dipolarquadrupolar fluids with various dipole and quadrupole moments were obtained by Dubey and O'Shea [14] from MC simulation. These workers found that increasing multipole strength will increase the critical temperature and broaden the vapor-liquid coexistence curves (the binodals). There have also been some theoretical studies aimed at understanding the mechanism of the formation of the spontaneous polarization at the surface of the dipolar-quadrupolar liquids
[15-17]. It was pointed out that this spontaneous polarization is responsible for the "sign effect" on the surface tension due to an external electric field [18] as well as the adsorption of ions with a preferred sign at the surface of the liquids [19]. For purely dipolar fluids, the spontaneous molecular orientation at the liquid surface has been studied on the basis of the integral equation [20] and " $f$-expansion" theory [21].

The density functional theory (DFT) is perhaps one of the most successful theoretical tools for studying vapor-liquid interfaces of polar fluids [2-4,20,22,23]. The DFT allows both the structural (the density and orientation profiles) and thermodynamical (surface tension) properties to be determined in a self-consistent way. For weakly dipolar fluids, the modified mean-field (MMF) DFT developed by Teixeira and co-workers $[2,4,24]$ has been employed by several workers $[25,26,9]$. A more sophisticated DFT has also been developed for strongly dipolar fluids $[3,23]$. In a previous paper [9] we employed the MMF-DFT to show that for a purely dipolar fluid subject to an external uniform electric field, changing the direction of the field has no effects on the surface tension. In a recent letter [27], we extended the MMFDFT to treat a dipolar-quadrupolar fluid. We find that changing the direction of the field does have an effect on the surface tension. We showed that for two given external fields with the same strength but exactly opposite direction, the magnitude of the field-induced change in the surface tension is different.

In this paper, we present full theoretical formalism of the extended MMF-DFT and detailed results of the properties of the dipolar-quadrupolar fluid and their dependence on the applied electric field. As noted in the previous letter [27], the study of the electric field effects on the interfacial properties of dipolar-quadrupolar fluids can be useful to our understanding of the sign-preference phenomenon observed in the ion-induced heterogeneous nucleation experiments [28-33] and computer simulation [34]. These experiments and computer simulation have shown that in the heterogeneous 
vapor-to-liquid nucleation on charged condensation centers the sign of the charge can affect appreciably the rate of nucleation. For example, it was found that water droplets nucleate faster on negative ions (anion preference) whereas methanol droplets nucleate faster on positive ions (cation preference), provided that the magnitude of all charges are identical. Several theoretical approaches have been developed to explain this phenomenon. Approaches include thermodynamics [35-37], MC [8] and molecular dynamics simulation $[38,39]$, and the DFT $[40,41]$. In essence, the sign-preference behavior can be attributed to certain asymmetry in molecular interaction. The dipolar-quadrupolar molecular model considered here does entail an intrinsic asymmetry of the charge distribution in the molecule. Thus, the dipolar-quadrupolar fluid should show sign preference in the ion-induced heterogeneous nucleation.

The rest of the paper is organized as follows. In Sec. II the MMF-DFT is extended to treat a dipolar-quadrupolar fluid subject to a uniform electric field. Results of bulk fluid properties are presented in Sec. III. Results of interfacial properties including the density and orientation order-parameter profiles, the surface polarization, and surface tension are shown in Sec. IV. The effects of the field direction on the order-parameter profiles and the surface tension are also discussed. Conclusions are given in Sec. V.

\section{DENSITY-FUNCTIONAL THEORY FOR DIPOLAR-QUADRUPOLAR FLUIDS}

We consider a single-component molecular fluid that consists of spherical particles each having a point dipole and a point quadrupole. In the body-fixed coordinate, the vector of the dipole is set to be the $z^{\prime}$ axis. The dipole thus has only one nonzero component $\mu_{z^{\prime}}=\mu_{0}$. For simplicity, we only consider that the quadrupole tensor has one nonzero component $Q_{z^{\prime} z^{\prime}}=Q$. The intermolecular interaction is described by a pairwise intermolecular potential $u\left(\vec{r}_{1}, \vec{r}_{2}, \omega_{1}, \omega_{2}\right)$, where $\vec{r}_{1}$ and $\vec{r}_{2}$ denote the positions of two molecules and $\omega_{1}$ and $\omega_{2}$ are the orientations of the molecular $z^{\prime}$ axes. We employed a perturbation approach to separate the intermolecular potential into two parts: a strongly repulsive interaction $u_{r e f}\left(\vec{r}_{1}, \vec{r}_{2}, \omega_{1}, \omega_{2}\right)$ and a relatively small perturbative attraction $u_{p e r}\left(\vec{r}_{1}, \vec{r}_{2}, \omega_{1}, \omega_{2}\right)=u\left(\vec{r}_{1}, \vec{r}_{2}, \omega_{1}, \omega_{2}\right)$ $-u_{r e f}\left(\vec{r}_{1}, \vec{r}_{2}, \omega_{1}, \omega_{2}\right)$. The former is treated as a reference system. Here, the reference potential is chosen to be the hard-sphere potential

$$
u_{r e f}\left(r_{12}\right)= \begin{cases}+\infty, & r_{12} \leqslant d \\ 0, & r_{12}>d\end{cases}
$$

and the perturbative potential is in the form

$$
\begin{aligned}
u_{p e r}\left(\vec{r}_{12}, \omega_{1}, \omega_{2}\right)= & {\left[u_{00}\left(r_{12}\right)+u_{d d}+u_{d q}+u_{q d}+u_{q q}\right] } \\
& \times H\left(r_{12}-d\right) .
\end{aligned}
$$

In Eqs. (1) and (2) $d$ is the hard-sphere diameter, $r_{12}=\left|\vec{r}_{12}\right|$ $=\left|\vec{r}_{2}-\vec{r}_{1}\right|$ is the intermolecular distance, and $H(r)$ is the
Heaviside step function. The isotropic part of the attractive interaction $u_{00}$ is taken to be the augmented Sutherland potential $[42,43]$

$$
u_{00}\left(r_{12}\right)=-4 \epsilon\left(d / r_{12}\right)^{6},
$$

where $\epsilon$ is the energy parameter. Such choices of separation of the reference and perturbative potentials have been used in many previous studies $[2,9,46-48]$. The choice of the Sutherland potential [Eq. (3)] as the isotropic part of the attractive potential is mainly for mathematical simplicity in the calculation of the interfacial properties of the polar fluid. Term $u_{d d}$ refers to the dipole-dipole interaction, i.e.,

$$
u_{d d}\left(\vec{r}_{12}, \omega_{1}, \omega_{2}\right)=\frac{\mu_{0}^{2}}{r_{12}^{3}}\left(c_{12}-3 c_{1} c_{2}\right),
$$

$u_{d q}+u_{q d}$ is the dipole-quadrupole interaction, i.e.,

$$
\begin{aligned}
u_{d q}\left(\vec{r}_{12}, \omega_{1}, \omega_{2}\right)+u_{q d}\left(\vec{r}_{12}, \omega_{1}, \omega_{2}\right) \\
=\frac{3}{2} \frac{\mu_{0} Q}{r_{12}^{4}}\left(c_{1}-c_{2}\right)\left(1+5 c_{1} c_{2}-2 c_{12}\right),
\end{aligned}
$$

and $u_{q q}$ is the quadrupole-quadrupole interaction [44], i.e.,

$$
\begin{aligned}
u_{q q}\left(\vec{r}_{12}, \omega_{1}, \omega_{2}\right)= & \frac{3}{4} \frac{Q^{2}}{r_{12}^{5}}\left(1-5 c_{1}^{2}-5 c_{2}^{2}+2 c_{12}^{2}\right. \\
& \left.+35 c_{1}^{2} c_{2}^{2}-20 c_{1} c_{2} c_{12}\right),
\end{aligned}
$$

where $c_{i}=\vec{n}_{i} \cdot \vec{n}, c_{12}=\vec{n}_{1} \cdot \vec{n}_{2}\left(\vec{n}_{1}\right.$ and $\vec{n}_{2}$ are the unit vectors along the molecular axes), and $\vec{n}=\vec{r}_{12} / r_{12}$ is the unit vector along the intermolecular axis.

Let $\rho(\vec{r}, \omega)=\rho(\vec{r}) \hat{f}(\vec{r}, \omega)$ denoting the number density of molecules, where $\rho(\vec{r})$ is the total number density of molecules without specifying the orientation and $\hat{f}(\vec{r}, \omega)$ denotes the distribution function of the molecular orientation $\omega$ $=(\theta, \varphi)$, with $\int d \omega \hat{f}(\vec{r}, \omega)=1$. The grand canonical potential of the system in the external field $V_{\text {ext }}(\vec{r}, \omega)$ can be expressed as $[2,25,23]$

$$
\begin{aligned}
\Omega[\rho(\vec{r}, \omega)]= & \int_{V} d \vec{r} f_{h s}(\rho(\vec{r}))+(1 / \beta) S+\Omega_{i n t} \\
& -\int_{V} d \vec{r} \int d \omega \rho(\vec{r}, \omega)\left[\mu-V_{\text {ext }}(\vec{r}, \omega)\right],
\end{aligned}
$$

where $\mu$ is the chemical potential of the system, $\beta$ $=1 /\left(k_{B} T\right)\left(k_{B}\right.$ is the Boltzman constant and $T$ is temperature), $f_{h s}(\rho(\vec{r}))$ is the free energy density of the hard-sphere reference system, and $V$ is the volume of the system. The term

$$
S=\int_{V} d \vec{r} \rho(\vec{r})\langle\ln [4 \pi \hat{f}(\vec{r}, \omega)]\rangle
$$


accounts for the loss of orientational entropy in the reference system, and $\langle\cdots\rangle \equiv \int d \omega \cdots \hat{f}(\vec{r}, \omega)$.

The orientation distribution function $\hat{f}(z, \omega)$ can be written as a sum of an isotropic part $1 / 4 \pi$ and a small anisotropic correction $\Delta \hat{f}(z, \omega)$ due to intermolecular and molecule-field interactions, i.e.,

$$
\hat{f}(\vec{r}, \omega)=1 / 4 \pi+\Delta \hat{f}(\vec{r}, \omega), \quad \Delta \hat{f} / \hat{f} \ll 1 .
$$

Keeping the terms up to $|\Delta \hat{f}|^{2}$, Eq. (8) can be rewritten as

$$
S=2 \pi \int_{V} d \vec{r} \rho(\vec{r}) \int d \omega|\Delta \hat{f}(\vec{r}, \omega)|^{2} .
$$

In Eq. (7), term $\Omega_{\text {int }}$ denotes the contribution due to the long-ranged perturbative part of the potential $u_{p e r}$ and thus it is dependent on the geometry of the dielectric system. A commonly used approximation to the pair correlation function that appears in the expression of $\Omega_{\text {int }}$ is the MMF approximation that approximates the correlation function by the Boltzmann factor $[2,25,23]$. As a result, $\Omega_{\text {int }}$ is given by

$$
\begin{aligned}
\Omega_{\text {int }}= & \frac{1}{2 \beta} \int_{V} d \vec{r}_{1} \int_{V} d \vec{r}_{2} \int d \omega_{1} d \omega_{2} \rho\left(\vec{r}_{1}, \omega_{1}\right) \rho\left(\vec{r}_{2}, \omega_{2}\right) \\
& \times e^{-\beta u_{r e f}\left(r_{12}\right)}\left\{1-\exp \left[-\beta u_{p e r}\left(\vec{r}_{12}, \omega_{1}, \omega_{2}\right)\right]\right\} .
\end{aligned}
$$

For weakly dipolar fluids, Teixeira and Telo da Gama [2] proposed to expand the second exponential term in Eq. (11) in powers of $\beta u_{p e r}$. If only the first-order term in the expansion is kept, it essentially gives the random phase approximation (RPA) to the correlation function. As shown in Refs. $[2,25]$ the RPA cannot account for the multipolar contribution to the bulk and interfacial properties of the fluid; the second-order terms in the expansion are needed for this purpose. Thus, in the present work, we also truncate the expansion after the second-order term. Finally, if the external electric field is uniform and has a magnitude $E, V_{e x t}$ is given by

$$
V_{\text {ext }}(\vec{r}, \omega)=-\mu_{0} E \cos \theta,
$$

where angle $\theta$ is measured relative to the field direction.

To describe the planar vapor-liquid interface, we consider a slab-shaped infinite system in which the $x-y$ plane is in parallel with the surfaces of the slab and the uniform electric field $\vec{E}$ is applied along the $z$ direction. Thus, the system is inhomogeneous only in the $z$ direction and the density profile $\rho(z)$ and the orientation distribution function $\hat{f}(z, \omega)$ depends only on the spatial variable $z$.

It has been shown previously [7] that in the presence of an external field the free energy of a homogeneous dipolar system depends on the shape of the system. Hence, we first consider that the system is contained in a finite cylindricalshape volume $V$. We then take the thermodynamical limit $V \rightarrow \infty$ such that the system assumes the slab shape. Note that the slab-shaped system considered here can be viewed as a planar capacitor subject to an electric field. A nice and simple feature of the planar capacitor is that the electric field depends only on the surface charge density of the two parallel plates, but not on the distance $L$ between the two plates. The expression for $\Omega_{\text {int }} / A$ in the thermodynamical limit can be written as

$$
\begin{aligned}
\lim _{V \rightarrow \infty} \frac{\Omega_{\text {int }}}{A}= & \lim _{\substack{L \rightarrow \infty \\
A \rightarrow \infty}} \frac{1}{2 A} \int_{-L / 2}^{L / 2} \int_{-L / 2}^{L / 2} d z_{1} d z_{2} \rho\left(z_{1}\right) \rho\left(z_{2}\right) \\
& \times \int d \omega_{1} d \omega_{2} \hat{f}\left(z_{1}, \omega_{1}\right) \hat{f}\left(z_{2}, \omega_{2}\right) \int_{A} \int_{A} d A_{1} d A_{2} \\
& \times\left(u_{\text {per }}-\frac{\beta}{2} u_{\text {per }}^{2}\right)\left(\vec{r}_{12}, \omega_{1}, \omega_{2}\right)
\end{aligned}
$$

where $A$ is the area of the cylinder base and $L$ is the axial length of the cylinder.

Substituting Eqs. (1)-(6) into Eq. (13), and carrying out the limit $A \rightarrow \infty$, we obtain the following form of $\Omega_{\text {int }} / A$ after a lengthy derivation (see Appendix A for mathematical details):

$$
\begin{aligned}
\lim _{V \rightarrow \infty} \frac{\Omega_{\text {int }}}{A}= & \frac{1}{2} \lim _{L \rightarrow \infty} \int_{-L / 2}^{L / 2} \int_{-L / 2}^{L / 2} d z_{1} d z_{2} \rho\left(z_{1}\right) \rho\left(z_{2}\right) \\
& \times \psi\left(z_{12}, \omega_{1}, \omega_{2}\right),
\end{aligned}
$$

where

$$
\begin{aligned}
\psi\left(z_{12}, \omega_{1}, \omega_{2}\right)= & \int d \omega_{1} d \omega_{2} \hat{f}\left(z_{1}, \omega_{1}\right) \hat{f}\left(z_{2}, \omega_{2}\right) \\
& \times \phi_{e f f}\left(z_{12}, \omega_{1}, \omega_{2}\right)
\end{aligned}
$$

and

$$
\begin{aligned}
\phi_{e f f}\left(z_{12}, \omega_{1}, \omega_{2}\right)= & \sum_{i, k=0(i \leqslant k)}^{4}\left[P_{i} P_{k}^{\prime} \phi_{i k}\left(z_{12}\right)\right. \\
& \left.+\left(1-\delta_{i, k}\right) P_{i}^{\prime} P_{k} \phi_{i k}\left(z_{21}\right)\right] .
\end{aligned}
$$

Here $\quad z_{12}=-z_{21}=z_{2}-z_{1}, \quad P_{i}=P_{i}\left(\cos \theta_{1}\right) \quad$ and $\quad P_{i}^{\prime}$ $=P_{i}\left(\cos \theta_{2}\right)$ are the Legendre polynomials of the $i$ th order, and $\delta_{i, k}$ is the Kronecker delta function. Functions $\phi_{i k}(z)$ are polynomials of $\mu_{0}$ and $Q$ up to the fourth order. The explicit expressions for $\phi_{i k}\left(z_{12}\right)$ are given by the Eq. (A24) in Appendix A. In the following section, we will discuss how to carry out the thermodynamical limit $L \rightarrow \infty$ in Eq. (14), for the bulk phases.

Since the planar system is invariant in the $x-y$ plane, the anisotropic part of the orientation distribution function $\Delta \hat{f}(z, \omega)$ is independent of the axial angle $\varphi$. Expanding $\Delta \hat{f}(z, \omega)$ in terms of the Legendre polynomial of $\cos \theta$ yields

$$
\Delta \hat{f}(z, \omega)=\frac{1}{4 \pi} \sum_{k=1}^{\infty}(2 k+1) \eta_{k}(z) P_{k}(\cos \theta),
$$

where the expansion coefficients 


$$
\eta_{k}(z)=\left\langle P_{k}(\cos \theta)\right\rangle \quad(k=1,2, \ldots)
$$

are the orientation order parameters. We also define $\eta_{0}=1$, which is consistent with Eqs. (9) and (18).

Inserting Eqs. (9), (12), and (17) into Eqs. (10) and (15) with using the orthogonality relation for the Legendre polynomials, the grand potential $[$ Eq. (7)] in the thermodynamical limit can be written as a functional of $\rho(z)$ and $\left\{\eta_{i}(z)\right\}$, i.e.,

$$
\begin{aligned}
& \Omega\left[\rho(z),\left\{\eta_{i}(z)\right\}\right] / A \\
& =\int_{-\infty}^{\infty} d z f_{h s}(\rho(z))+\frac{1}{2 \beta} \int_{-\infty}^{\infty} d z \rho(z) \sum_{i=1}^{\infty}(2 i+1) \eta_{i}^{2}(z) \\
& \quad+\frac{1}{2} \int_{-\infty}^{\infty} \int_{-\infty}^{\infty} d z_{1} d z_{2} \psi\left(z_{1}, z_{2}\right) \rho\left(z_{1}\right) \rho\left(z_{2}\right) \\
& \quad-\int_{-\infty}^{\infty} d z \mu \rho(z)-\int_{-\infty}^{\infty} d z \mu_{0} E \rho(z) \eta_{1}(z),
\end{aligned}
$$

where

$$
\begin{aligned}
\psi\left(z_{1}, z_{2}\right)= & \sum_{i, k=0(i \leqslant k)}^{4}\left[\eta_{i}\left(z_{1}\right) \eta_{k}\left(z_{2}\right) \phi_{i k}\left(z_{12}\right)\right. \\
& \left.+\left(1-\delta_{i, k}\right) \eta_{k}\left(z_{1}\right) \eta_{i}\left(z_{2}\right) \phi_{i k}\left(z_{21}\right)\right] .
\end{aligned}
$$

Applying the variational principle to the grand potential [Eq. (19)] with respect to $\rho\left(z_{1}\right)$ and $\left\{\eta_{i}\left(z_{1}\right)\right\}$ yields a set of coupled integral equations:

$$
\begin{aligned}
\mu=\mu_{h s}\left(\rho\left(z_{1}\right)\right) & +\frac{1}{2 \beta} \sum_{i=1}^{4}(2 i+1) \eta_{i}^{2}\left(z_{1}\right)-\mu_{0} E \eta_{1}\left(z_{1}\right) \\
+\int_{-\infty}^{\infty} d z_{2} \psi\left(z_{1}, z_{2}\right) \rho\left(z_{2}\right) & \\
\eta_{i}\left(z_{1}\right)= & \delta_{i, 1} \frac{1}{3} \beta \mu_{0} E-\frac{1}{(2 i+1)} \beta \int_{-\infty}^{\infty} d z_{2} \\
& \times\left[\sum_{k=0(i \leqslant k)}^{4} \phi_{i k}\left(z_{12}\right) \eta_{k}\left(z_{2}\right)\right. \\
& \left.+\sum_{k=0(i>k)}^{4} \phi_{i k}\left(z_{21}\right) \eta_{k}\left(z_{2}\right)\right] \\
& \times \rho\left(z_{2}\right)
\end{aligned}
$$

and

$$
\eta_{i}\left(z_{1}\right)=0 \quad(i>4)
$$

where $\mu_{h s}(\rho(z))$ is the local chemical potential of the hardsphere fluid, which can be accurately evaluated by using the Carnahan-Starling formula [45].

\section{BULK FLUID PROPERTIES}

For bulk phases, density $\rho$ and the order parameters $\eta_{1}$, $\eta_{2}, \eta_{3}$, and $\eta_{4}$ are constant. The expression for the interaction part of the grand canonical potential $\Omega_{i n t} / V$ in the thermodynamical limit can be written, on the basis of Eqs. (14) and (20), as

$$
\lim _{V \rightarrow \infty} \frac{\Omega_{i n t}}{V}=\frac{\rho^{2}}{2} \lim _{L \rightarrow \infty} \frac{1}{L} \int_{-L / 2}^{L / 2} \int_{-L / 2}^{L / 2} d z_{1} d z_{2} \psi\left(z_{12}\right)
$$

where

$$
\psi\left(z_{12}\right)=\sum_{i, k=0(i \leqslant k)}^{4}\left[\phi_{i k}\left(z_{12}\right)+\left(1-\delta_{i, k}\right) \phi_{i k}\left(z_{21}\right)\right] \eta_{i} \eta_{k} .
$$

With the new variables $z_{s}=\left(z_{1}+z_{2}\right) / 2$ and $z_{12}=z_{2}-z_{1}$ the integral on the right-hand side of Eq. (24) can be transformed as follows:

$$
\begin{aligned}
\int_{-L / 2}^{L / 2} \int_{-L / 2}^{L / 2} d z_{1} d z_{2} \psi\left(z_{12}\right) & =\int_{-L / 2}^{L / 2} d z_{s} \int_{-L}^{L} d z_{12} \psi\left(z_{12}\right) \\
& =L \int_{-L}^{L} d z_{12} \psi\left(z_{12}\right)
\end{aligned}
$$

Using this expression, after carrying out the limit $L \rightarrow \infty$, Eq. (24) can be written as

$$
\lim _{V \rightarrow \infty} \frac{\Omega_{i n t}}{V}=\frac{\rho^{2}}{2} \sum_{i, k=0(i \leqslant k)}^{4}\left[1+(-1)^{(i+k)}-\delta_{i, k}\right] \Phi_{i k} \eta_{i} \eta_{k}
$$

where

$$
\Phi_{i k}=\int_{-\infty}^{\infty} \phi_{i k}(z) d z
$$

All $\Phi_{i k}$ are finite, because for any indexes $i$ and $k$ with $z$ $\rightarrow \infty$ the integrand in Eq. (28) converges to zero as $\phi_{i k}$ $\rightarrow 1 / z^{n}(n \geqslant 4)$ (see Appendix A). Finally, in the thermodynamical limit the expression for $\Omega / V$ of the bulk can be written as

$$
\begin{aligned}
\lim _{V \rightarrow \infty} \frac{\Omega}{V}= & f_{h s}(\rho)+\frac{1}{2 \beta} \rho \sum_{i=1}^{4}(2 i+1) \eta_{i}^{2}+\frac{\rho^{2}}{2} \\
& \times \sum_{i, k=0(i \leqslant k)}^{4}\left[1+(-1)^{(i+k)}-\delta_{i, k}\right] \Phi_{i k} \eta_{i} \eta_{k} \\
& -\mu \rho-\mu_{0} E \rho \eta_{1} .
\end{aligned}
$$

We provide detailed expressions for all $\Phi_{i k}$ in Appendix B. Moreover, noting that $\Phi_{01}, \Phi_{02}, \Phi_{03}, \Phi_{04}, \Phi_{12}, \Phi_{14}$, $\Phi_{23}, \Phi_{24}$, and $\Phi_{34}$ are all equal to zero [see Eq. (B1)], the minimization of $\lim _{V \rightarrow \infty} \Omega / V$ [Eqs. (29)] with respect to $\left\{\eta_{i}\right\}$ yields the order parameters 


$$
\eta_{1}=\frac{\frac{\mu_{0} E}{3 k_{B} T}}{1+\frac{\rho \Phi_{11}}{3 k_{B} T}}, \quad \eta_{3}=-\eta_{1} \frac{\frac{\Phi_{13} \rho}{7 k_{B} T}}{\left(1+\frac{\Phi_{33} \rho}{7 k_{B} T}\right)}
$$

and

$$
\eta_{2}=0, \quad \eta_{4}=0
$$

With these results, after the minimization of $\lim _{V \rightarrow \infty} \Omega / V$ with respect to $\rho$, the chemical potential of the bulk fluid can be written as

$$
\frac{\mu}{k_{B} T}=\frac{\mu_{h}(\rho)}{k_{B} T}-\frac{3}{2} \eta_{1}^{2}-\frac{7}{2} \eta_{3}^{2}+\frac{\rho}{k_{B} T} \Phi_{00} .
$$

Using Eqs. (30)-(32) the grand potential of the bulk fluid [Eq. (29)] in the thermodynamical limit can be written as

$$
\begin{aligned}
\frac{\Omega}{V}= & -p_{h}(\rho)-\frac{1}{2 k_{B} T} \rho^{2}\left(\Phi_{00}+\eta_{1}^{2} \Phi_{11}+\eta_{3}^{2} \Phi_{33}\right. \\
& \left.+2 \eta_{1} \eta_{3} \Phi_{13}\right),
\end{aligned}
$$

where $p_{h}=\rho \mu_{h}-f_{h}$ is the hard-sphere pressure. At a given temperature $T$, the coexisting vapor-liquid densities $\rho_{l}$ and $\rho_{v}$ can be calculated by finding the simultaneous solution of the grand potential equation $\Omega\left(\rho_{l}, T\right)=\Omega\left(\rho_{v}, T\right)$ and the chemical potential equation $\mu\left(\rho_{l}, T\right)=\mu\left(\rho_{v}, T\right)$.

\section{A. Zero field}

In the zero field $(E=0)$, it follows from Eq. (30) that the order parameters $\eta_{1}$ and $\eta_{3}$ become zero. This is consistent with the fact that there is no favorable orientation for the dipolar-quadrupolar molecules in the bulk fluid phase. Hereafter, we will use dimensionless quantity $\mu_{0}^{*}=\mu_{0} /\left(\epsilon d^{3}\right)^{1 / 2}$ for the dipole moment, $Q^{*}=Q /\left(\epsilon d^{5}\right)^{1 / 2}$ for the quadrupole moment, $T^{*}=k_{B} T / \epsilon$ for the temperature, $\eta=\left(\pi d^{3} / 6\right) \rho$ for the density, and $E^{*}=E\left(d^{3} / \epsilon\right)^{1 / 2}$ for the electric field. Because the MMF-DFT is only applicable for weakly dipolarquadrupolar fluids, the values of multipolar moment are chosen within the range $0 \leqslant \mu_{0}^{*} \leqslant 1$ and $0 \leqslant Q^{*} \leqslant 1$. In Fig. 1 , we plot the vapor-liquid coexistence curves for various given $\mu_{0}^{*}$ and $Q^{*}$. One can see that for a given $T^{*}$, increasing $\mu_{0}^{*}$ or $Q^{*}$ broadens the coexistence curves, decreases the vapor density $\eta_{v}$, and increases the liquid density $\eta_{l}$. For the given $\left(\mu_{0}^{*}, Q^{*}\right)=(1,0),(0,1),(1,0.8),(0.8,1)$, and $(1,1)$ the critical temperatures $T_{c}^{*}$ is $2.06,2.10,2.15,2.17$, and 2.22 , respectively. It is found that the difference in the critical temperature between the polar fluid and the nonpolar counterpart can be approximately correlated with the relation

$$
T_{c}^{*}\left(\mu_{0}^{*}, Q^{*}\right)-T_{c}^{*}(0,0) \sim \mu_{0}^{* 4}+\frac{9}{5} \mu_{0}^{* 2} Q^{* 2}+\frac{9}{5} Q^{* 4},
$$

and the correlation is better for smaller values of $\mu_{0}^{*}$ and $Q^{*}$. Obviously, the right-hand side of Eq. (34) stems from those terms having multipolar moment dependence in the grand potential [Eq. (33)]. Equation (34) indicates that the

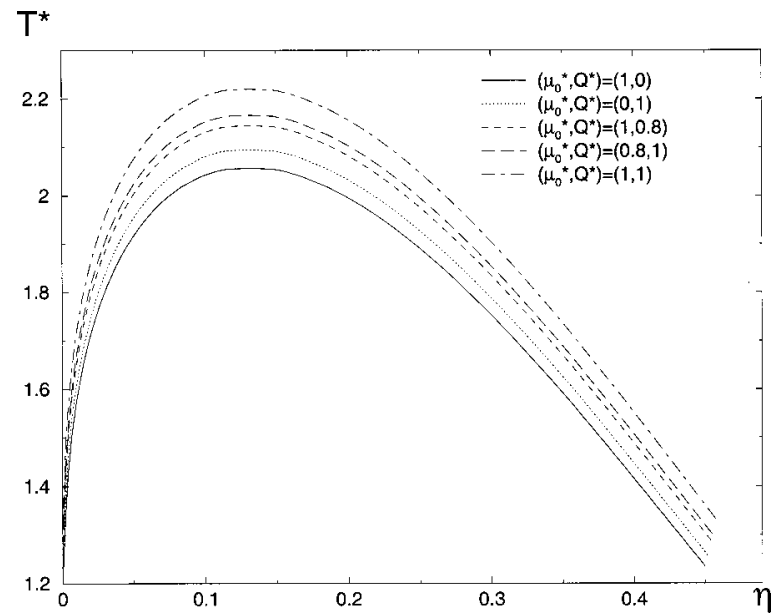

FIG. 1. Vapor-liquid coexistence densities for the dipolarquadrupolar fluid with various given multipolar moments $\left(\mu_{0}^{*}, Q^{*}\right)$.

critical temperature difference grows faster with increasing $Q^{*}$ than increasing $\mu_{0}^{*}$. Indeed, the fact that the critical temperature for the fluid with $\left(\mu_{0}^{*}, Q^{*}\right)=(0.8,1)$ is higher than that with $\left(\mu_{0}^{*}, Q^{*}\right)=(1,0.8)$ confirms that $T_{c}^{*}$ has a stronger dependence on $Q^{*}$ than on $\mu_{0}^{*}$. This can be understood on the basis of the contribution of the attraction energy $\Phi_{00}[\mathrm{Eq}$. (B2)] to the grand potential [Eq. (33)]: the contribution of dipolar interaction is $\left(\mu_{0}^{*}\right)^{4} / 12$, whereas the contribution of quadrupolar interaction is $(3 / 20)\left(Q_{0}^{*}\right)^{4}$ (in addition to the contribution due to the dipolar-quadrupolar interaction to $\left.\Phi_{00}\right)$. Given the same dimensionless values of $\mu_{0}^{*}$ and $Q^{*}$ the quadrupolar interaction is $9 / 5$ time larger than the dipolar interaction. Note that for the limiting case of purely dipolar $\left(Q^{*}=0\right)$ and purely quadrupolar fluid $\left(\mu_{0}^{*}\right.$ $=0)$, Eq. (34) reduces to the known relation $[2,23,46]$ $T_{c}^{*}\left(\mu_{0}^{*}\right)-T_{c}^{*}(0) \sim \mu_{0}^{* 4}$ and $T_{c}^{*}\left(Q^{*}\right)-T_{c}^{*}(0) \sim Q^{* 4}$, respectively.

Our results are in qualitative agreement with the MC simulation of the LJ dipolar-quadrupolar fluids by Dubey and O'Shea [14]. For the given values of dipole and quadrupole moments, the critical temperatures determined from the $\mathrm{MC}$ simulation are given as follows [14]: $T_{c}^{*}\left[\left(\mu_{0}^{*}, Q^{*}\right)=(1,0)\right]$ $=1.41, \quad T_{c}^{*}\left[\left(\mu_{0}^{*}, Q^{*}\right)=(0,1)\right]=1.60$, and $T_{c}^{*}\left[\left(\mu_{0}^{*}, Q^{*}\right)\right.$ $=(1,1)]=1.79$, which indicate that $T_{c}^{*}$ has a stronger dependence on $Q^{*}$ than on $\mu_{0}^{*}$. Furthermore, Dubey and O'Shea showed that for $\mu_{0}^{*}=1$, increasing $Q^{*}$ from 0 to 1 raises $T_{c}^{*}$ by $27 \%$, whereas for $Q^{*}=1$, increasing $\mu_{0}^{*}$ from 0 to 1 only raises $T_{c}^{*}$ by $12 \%$. They [14] also plotted $T_{c}^{*}$ versus $Q^{* 2}$ for $\mu_{0}^{* 2}=1$ and $Q^{* 2}=1.0,1.5,2.0,2.5$, and found a linear relation between $T_{c}^{*}$ and $Q^{* 2}$, which is consistent with our result [Eq. (34)], but only for $Q^{* \ll 1}$. It appears to us that some points in Fig. 7 of Ref. [14] could be also connected via a parabola, which would agree better with our results for higher values of $Q^{*}$.

We also examined whether the coexistence densities satisfy the law of corresponding states. In Fig. 2, the coexistence curves in Fig. 1 are replotted with a rescaled axis $T^{*} / T_{c}^{*}\left(\mu_{0}^{*}, Q^{*}\right)$. It can be seen that the curves nearly col- 


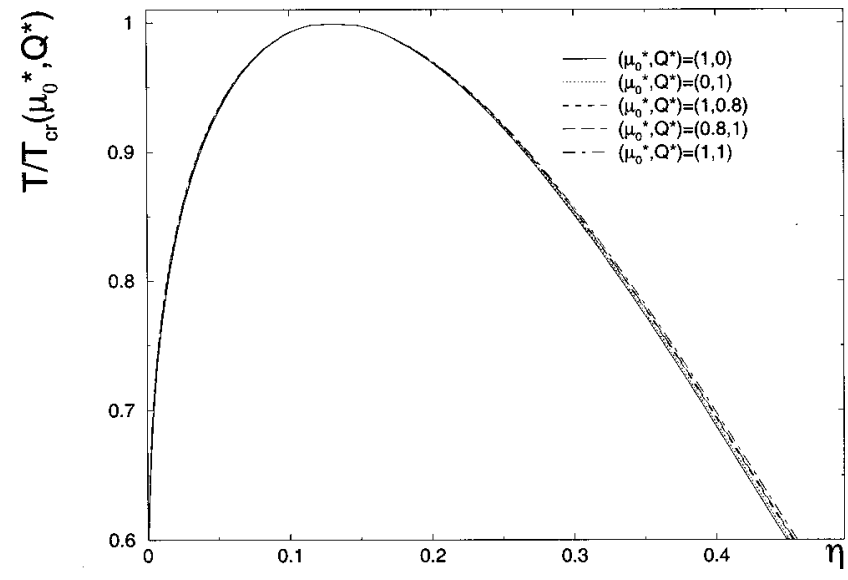

FIG. 2. The same as Fig. 1 except the temperature is rescaled based on the critical temperature.

lapse onto a single master curve (except at the high densities), indicating that the law of corresponding states is largely satisfied for weakly dipolar-quadrupolar fluids. This satisfaction of the law of corresponding states was also seen in the MC simulation of dipolar-quadrupolar fluids (see Figs. 8 and 9 of Ref. [14]). Moreover, we find that near the critical point the difference in the coexistence liquid and vapor densities also satisfies the mean-field scaling relation

$$
\eta_{l}-\eta_{v} \sim \tau^{1 / 2}
$$

where $\tau \equiv 1-T / T_{c}\left(\mu_{0}^{*}, Q^{*}\right)$.

\section{B. Nonzero field}

Here we consider the effects of a uniform electric field on the vapor-liquid coexistence for $\left(\mu_{0}^{*}, Q^{*}\right)=(1,0.8)$. In Fig. 3 , we plot the vapor-liquid coexistence curves for two given magnitudes of the electric field $E^{*}=0.2$ and 0.5. Compared to the zero-field curves, it can be seen that increasing the magnitude of the electric field narrows the coexistence curves, enlarges the coexistence vapor density, and reduces the coexistence liquid density. This behavior is opposite to that due to increasing the multipolar moments. As shown in the preceding section, in the zero field all the order parameters are zero. In contrast, in a nonzero field the order parameters $\eta_{1}$ and $\eta_{3}$ became finite [Eq. (30)]. It is found that $\eta_{1}$ is more sensitive to change in the magnitude of the electric field (Fig. 4) compared to $\eta$. Note that in Fig. 4, the larger values of $\eta_{1}$ correspond to the coexisting vapor phase and smaller values correspond to the liquid phase [9]. Note also that although the coexistence density curves are not affected by changing the direction of the field, $\eta_{1}$ does depend on the field direction. The order parameter $\eta_{3}$ is not plotted here since it is about two orders of magnitude smaller than $\eta_{1}$. Finally, we also find that near the critical point not only the coexistence-density difference satisfies the mean-field scaling relation Eq. (35), but also the difference in the order parameter $\eta_{1 v}$ and $\eta_{1 l}$ at phase coexistence satisfies a similar relation for the given values of $E^{*}$ :

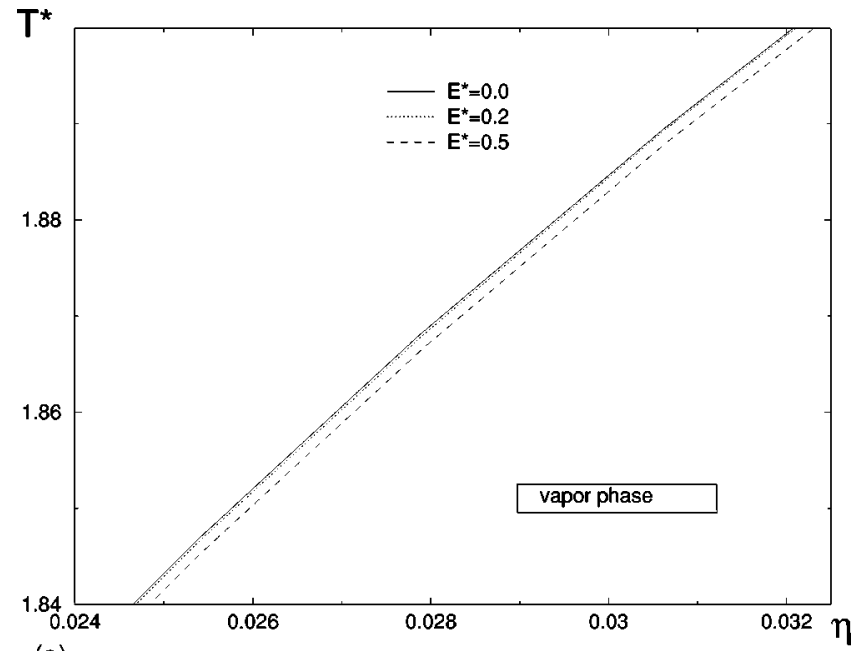

(a)

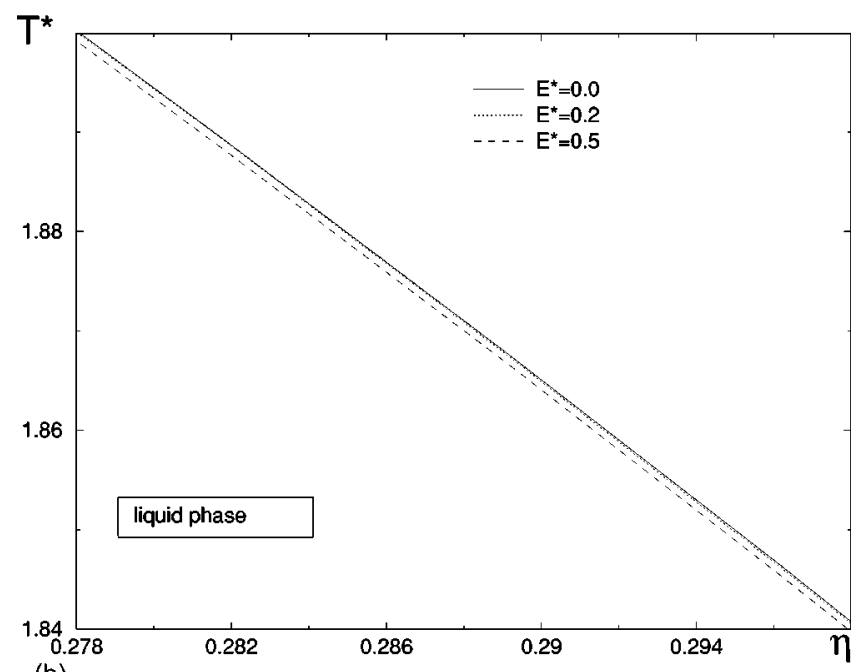

(b)

FIG. 3. The vapor-liquid coexistence curves for $\left(\mu_{0}^{*}, Q^{*}\right)$ $=(1,0.8)$ and given electric field $E^{*}=0.2 ; 0.5$ : (a) the vapor branch and (b) the liquid branch.

$$
\eta_{1 v}-\eta_{1 l} \sim \tau^{1 / 2},
$$

where $\tau=1-T / T_{c}\left(\mu_{0}^{*}, Q^{*}, E^{*}\right)$. The difference in the coexistence densities $\eta_{l}$ and $\eta_{v}$ in the electric field still obeys the power law of Eq. (35).

\section{PLANAR INTERFACIAL PROPERTIES}

The density and orientation order-parameter profiles of the planar vapor-liquid interface can be calculated by numerically solving the integral equations (21) and (22) using the iterative method [9]. Generally, the initial input for the iteration is taken to be a step function having the bulk coexistence values. However, we found that the iteration becomes unstable for low values of $T^{*}$. This numerical problem becomes more serious if the $\mu^{*}$ or $Q^{*}$ value is large. The nature of this iteration instability at low values of $T^{*}$ has 


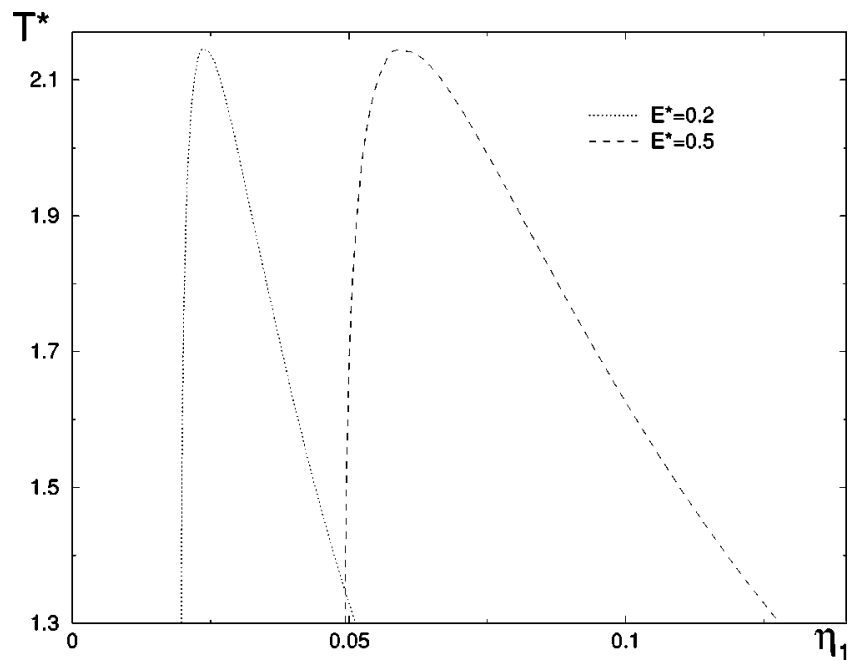

FIG. 4. The orientation order parameter $\eta_{1}$ at vapor-liquid coexistence for $\left(\mu_{0}^{*}, Q^{*}\right)=(1,0.8)$ and $E^{*}=0.2,0.5$. At a fixed $E^{*}$ the branch with the larger values of $\eta_{1}$ corresponds to the vapor phase and the branch with the smaller values of $\eta_{1}$ corresponds to the liquid phase.

been previously discussed in Ref. [47]. It appears to be a specific problem associated with the MMF-DFT.

It has been shown that for purely dipolar [20,2,23] or purely quadrupolar fluids in the zero field $[21,46]$, the lowest nonzero order-parameter profile is $\eta_{2}(z)$. In a nonzero field, however, the lowest nonzero order-parameter profile is $\eta_{1}(z)$ for purely dipolar fluid [9]. On the other hand, for the dipolar-quadrupolar fluid the lowest nonzero order-parameter profile is still $\eta_{1}(z)$ even in the zero field. This can be seen from Eq. (22) whose solution gives the order-parameter profiles $\eta_{1}(z), \eta_{2}(z), \eta_{3}(z)$, and $\eta_{4}(z)$, assuming that the functions $\phi_{01}, \phi_{02}, \phi_{03}$, and $\phi_{04}$ appearing in the integrals are nonzero. In particular, $\phi_{01}$ contains terms $\chi_{d d, d q}^{01}$ and $\chi_{d q, q q}^{01}$ [Eq. (A24)], which account for the coupling effect of dipoles and quadrupoles, i.e., $\chi_{d d, d q}^{01} \sim \mu_{0}^{3} Q[\mathrm{Eq} .(\mathrm{A} 21)]$ and $\chi_{d q, q q}^{01} \sim \mu_{0} Q^{3}$ [Eq. (A23)]. If either $\mu_{0}$ or $Q$ is zero, both $\chi_{d d, d q}^{01}$ and $\chi_{d q, q q}^{01}$ will be zero and so will $\phi_{01}$. This explains why the order-parameter profile $\eta_{1}(z)$ is zero for purely dipolar or quadrupolar fluids. On the other hand, function $\phi_{02}$ in the integral equation for $\eta_{2}(z)$ [Eq. (22)] is a sum of functions $D, E, F, \chi_{d q, d q}^{02}$, and $\chi_{q q, q q}^{02}[$ see Eq. (A24)]. Since $D, E$, and $F \sim \mu_{0}^{4}, \quad \chi_{q q, q q}^{02} \sim Q^{4}$ (see Appendix A), and $\chi_{d q, d q}^{02} \sim \mu_{0}^{2} Q^{2}$ [Eq. (A19)], if $\mu_{0}$ or $Q$ is nonzero $\phi_{02}$ will be nonzero, and so will the order-parameter profile $\eta_{2}(z)$.

\section{A. Zero field}

First, we consider the temperature dependence of the interfacial profiles. In Figs. 5(a)-5(c), we plot the number density and order-parameter profiles for $\left(\mu_{0}^{*}, Q^{*}\right)=(1,1)$ and various given $\tau$. Figure 5(a) shows that the density profile $\eta(z)$ is broadened with decreasing $\tau$. For $\tau \ll 1$ the interfacial 10-90 width $W$ diverges as

$$
W \sim \tau^{-1 / 2}
$$

$\eta$

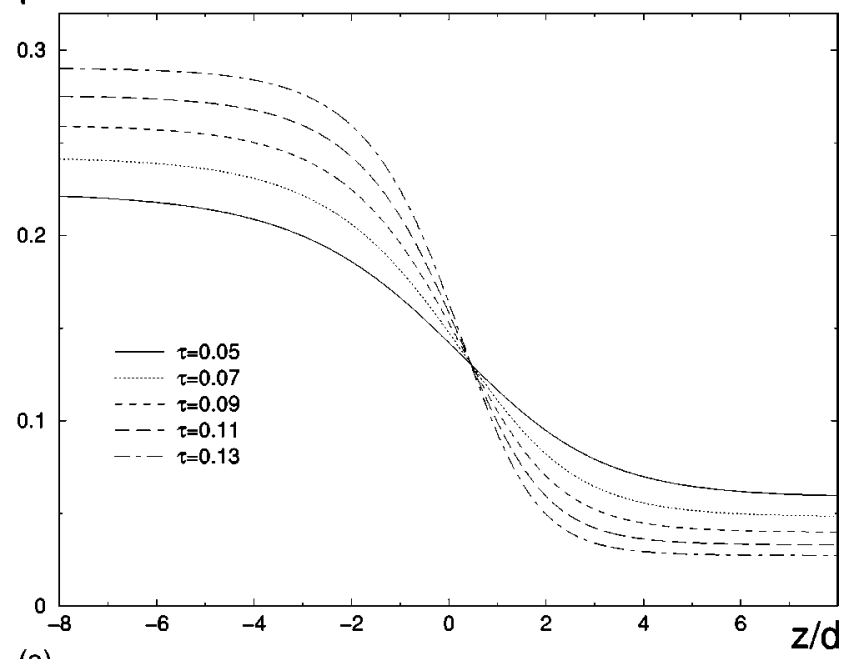

(a)

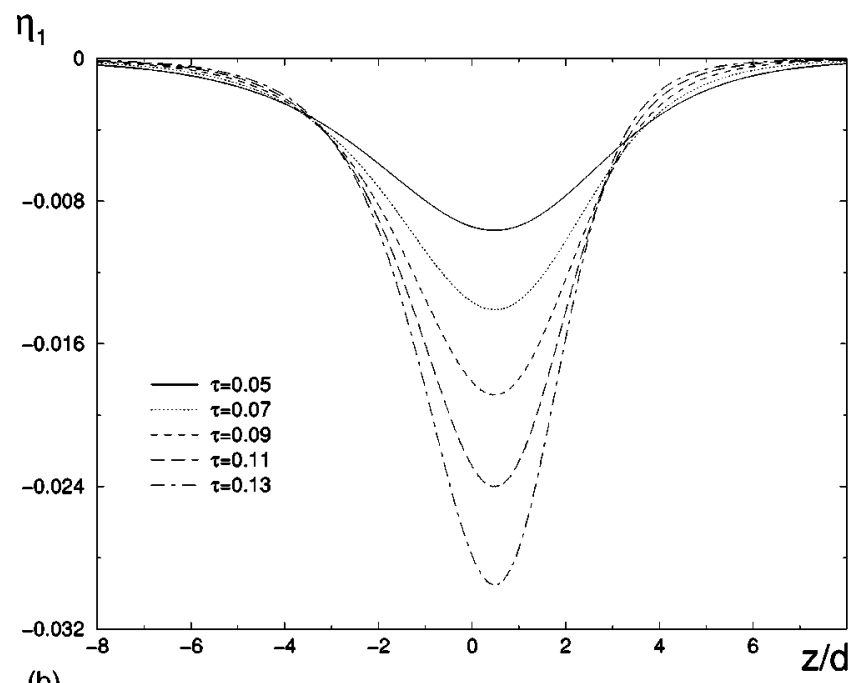

(b)

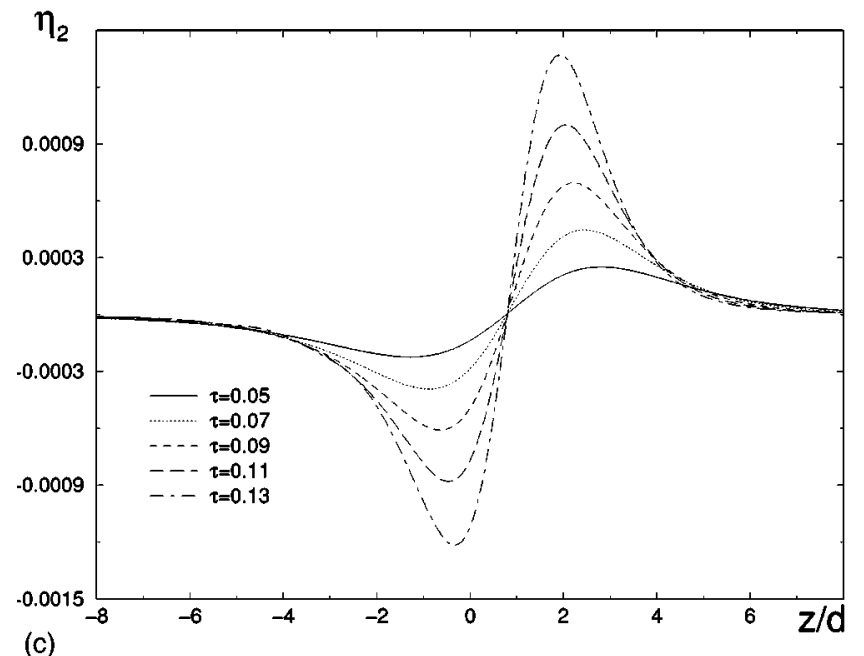

FIG. 5. (a) The density profile $\eta(z)$, and order-parameter profile (b) $\eta_{1}(z)$ and (c) $\eta_{2}(z)$ for $\left(\mu_{0}^{*}, Q^{*}\right)=(1,1)$ and various given $\tau$ $\equiv 1-T / T_{c}\left(\mu_{0}^{*}, Q^{*}\right)$. 
Figures 5(b) and 5(c) show that the two leading orientation order-parameter profiles, $\eta_{1}(z)$ and $\eta_{2}(z)$, are flattened and broadened with decreasing $\tau$. Recall that $\eta_{1}$ and $\eta_{2}$ are defined as $\langle\cos \theta\rangle$ and $\left\langle P_{2}(\cos \theta)\right\rangle$, respectively [Eq. (18)]. The fact that $\eta_{1}(z)$ is negative across the interface indicates that dipoles at the interface tend to align themselves pointing towards the liquid [47]; the fact that $\eta_{2}(z)$ is negative on the liquid side but positive on the vapor side indicates that molecules tend to orient their molecular axis in parallel with the interface on the liquid side but normal to the interface on the vapor side $[2,23]$.

As a measure of the interfacial orientation order on the basis of $\eta_{1}(z)$, we define parameter $D_{\eta_{1}} \equiv\left|\min \left(\eta_{1}\right)\right|$. We find that for small $\tau, D_{\eta_{1}}$ scales with $\tau$ as

$$
D_{\eta_{1}} \sim \tau \text {. }
$$

Denoting the position of the minimum of $\eta_{1}(z)$ to be $z_{\min }^{(1)}$, i.e., $\min \eta_{1}(z)=\eta_{1}\left(z_{\min }^{(1)}\right)$, the width of the $\eta_{1}(z)$ profile can be defined as $W_{\eta_{1}}=z_{2}^{(1)}-z_{1}^{(1)}$, where $z_{1}^{(1)}$ and $z_{2}^{(1)}$ are defined from equation $\eta_{1}\left(z_{2}^{(1)}>z_{\min }^{(1)}\right)=\eta_{1}\left(z_{1}^{(1)}<z_{\min }^{(1)}\right)=0.1 \eta_{1}\left(z_{\min }^{(1)}\right)$. As such, we find that $W_{\eta_{1}}$ diverges with $\tau$ as

$$
W_{\eta_{1}} \sim \tau^{-1 / 2} \text {. }
$$

The interfacial orientation order measured on the basis of $\eta_{2}(z)$ can be given by the difference $D_{\eta_{2}}=\max \left(\eta_{2}\right)$ $-\min \left(\eta_{2}\right)$. We find that $D_{\eta_{2}}$ scales with $\tau$ as

$$
D_{\eta_{2}} \sim \tau^{3 / 2} .
$$

Denoting the positions of the maximum and minimum of $\eta_{2}(z)$ to be $z_{\max }^{(2)}$ and $z_{\min }^{(2)}$, i.e., $\max \eta_{2}=\eta_{2}\left(z_{\max }^{(2)}\right)$ and $\min \eta_{2}$ $=\eta_{2}\left(z_{\min }^{(2)}\right)$, the width $W_{\eta_{2}}$ of the $\eta_{2}(z)$ profile can be defined by $z_{2}^{(2)}-z_{1}^{(2)}$, where $\eta_{2}\left(z_{1}^{(2)}<z_{\min }^{(2)}\right)=0.1 \eta_{2}\left(z_{\min }^{(2)}\right) \quad$ and $\eta_{2}\left(z_{2}^{(2)}>z_{\max }^{(2)}\right)=0.1 \eta_{2}\left(z_{\max }^{(2)}\right)$. We find that width $W_{\eta_{2}}$ scales with $\tau$ as

$$
W_{\eta_{2}} \sim \tau^{-1 / 2}
$$

The mean-field scaling relations, Eqs. (40) and (41), have been previously obtained for purely dipolar fluid [3] as well as for purely quadrupolar fluid [46]. To summarize, all the scaling relations Eqs. (37)-(41) seem to hold for all the considered $\mu_{0}^{*}$ and $Q^{*}$, and they hold particularly well for smaller values of $\mu_{0}^{*}$ and $Q^{*}$.

Second, we consider the multipole moment dependence of the interfacial profiles at a fixed temperature. In Fig. 6(a) we show the density profiles $\eta(z)$ for $T^{*}=1.95$ and various given $\left(\mu_{0}^{*}, Q^{*}\right)$. It can be seen that increasing the multipole moments makes the density profile steeper and reduces the surface thickness. In the second column of Table I we show the numerical values of the 10-90 interfacial width for the given $\left(\mu_{0}^{*}, Q^{*}\right)$. We find that for $\mu_{0}^{*}=1$, increasing $Q^{*}$ from 0 to 1 reduces $W$ by $41 \%$, but for $Q^{*}=1$ increasing $\mu_{0}^{*}$ from 0 to 1 reduces $W$ only by $30 \%$. Figures $6(\mathrm{~b})$ and 6(c) show the the orientation order-parameter profiles $\eta_{1}(z)$

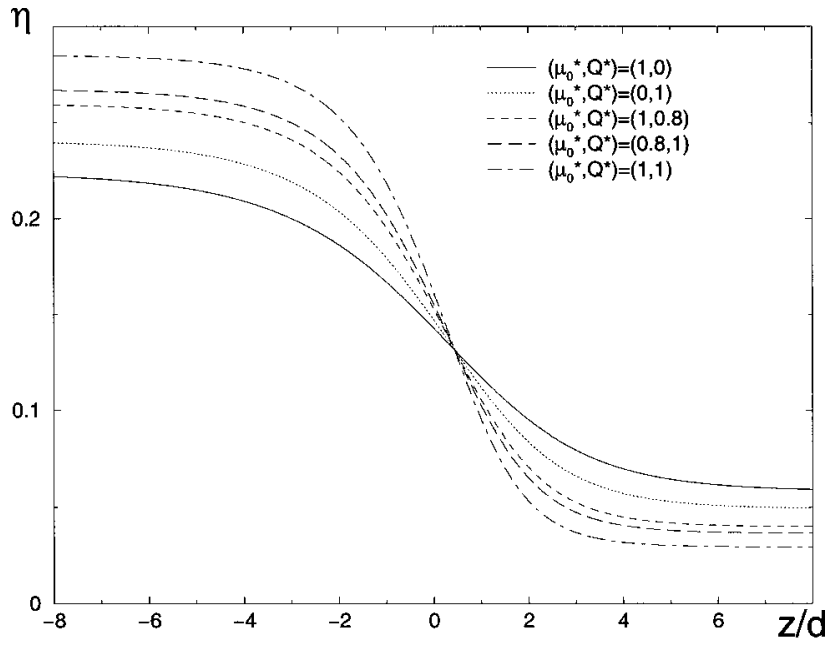

(a)

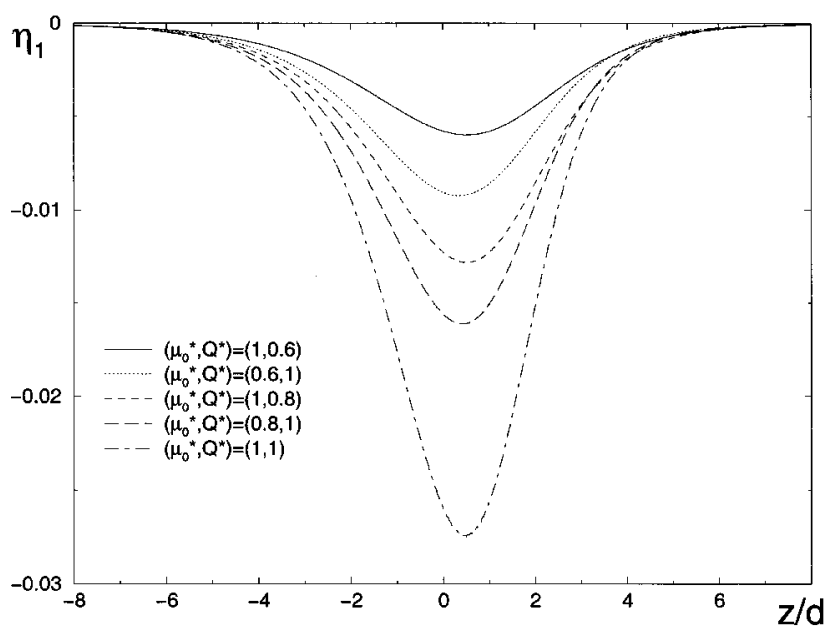

(b)

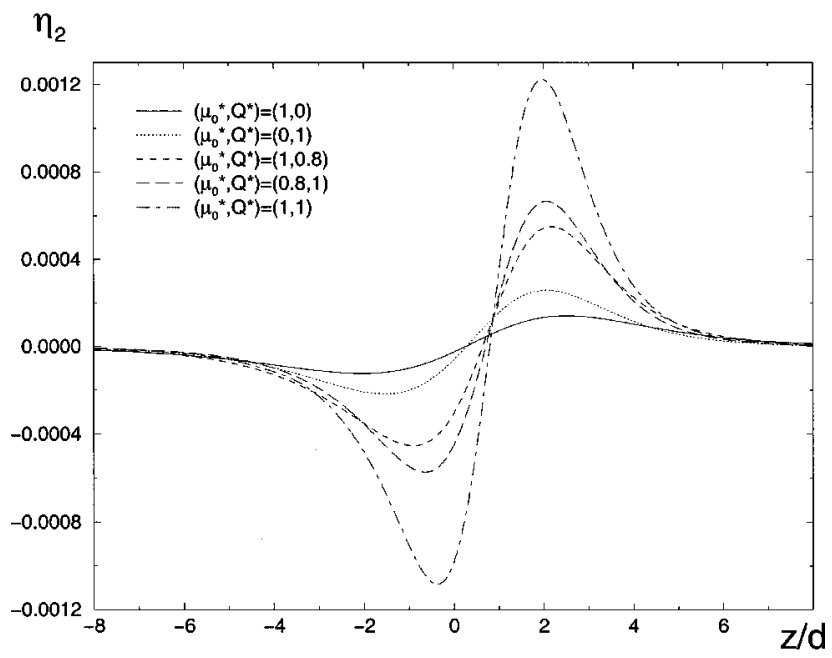

(c)

FIG. 6. (a) The density profile $\eta(z)$, and order-parameter profile (b) $\eta_{1}(z)$ and (c) $\eta_{2}(z)$ for $T^{*}=1.95$ and various given $\left(\mu_{0}^{*}, Q^{*}\right)$. 
TABLE I. The 10-90 width of the interface $W / d$, the degree of orientation order $D_{\eta_{1}}$, the width of $\eta_{1}(z)$ profile $W_{\eta_{1}} / d$, the degree of orientation order $D_{\eta_{2}}$, and the width of $\eta_{2}(z)$ profile $W_{\eta_{2}} / d$ for $T^{*}=1.95$, and various given $\left(\mu_{0}^{*}, Q^{*}\right)$.

\begin{tabular}{cccccc}
\hline \hline$\left(\mu_{0}^{*}, Q^{*}\right)$ & $W / d$ & $D_{\eta_{1}}$ & $W_{\eta_{1}} / d$ & $D_{\eta_{2}}\left(\times 10^{-3}\right)$ & $W_{\eta_{2}} / d$ \\
\hline$(1,0)$ & 7.19 & 0.00 & & 0.26 & 16.69 \\
$(0,1)$ & 6.03 & 0.00 & & 0.47 & 12.72 \\
$(1,0.8)$ & 5.14 & 1.28 & 8.79 & 1.01 & 11.63 \\
$(0.8,1)$ & 4.81 & 1.61 & 8.27 & 1.24 & 11.06 \\
$(1,1)$ & 4.23 & 2.74 & 7.36 & 2.31 & 8.93 \\
\hline \hline
\end{tabular}

and $\eta_{2}(z)$ for $T^{*}=1.95$ and the same given $\left(\mu_{0}^{*}, Q^{*}\right)$. Increasing the multipole moments increases the orientation orders $D_{\eta_{1}}$ and $D_{\eta_{2}}$, and reduces the width $W_{\eta_{1}}$ and $W_{\eta_{2}}$. One can also see in Table I that the values of $D_{\eta_{1}}, D_{\eta_{2}}, W_{\eta_{1}}$, and $W_{\eta_{2}}$ have a stronger dependence on $Q^{*}$ than on $\mu_{0}^{*}$. Finally, we find that the even-numbered order parameters $\eta_{2}(z)$ and $\eta_{4}(z)$ are independent of the sign of $Q^{*}$, whereas the oddnumbered order parameters $\eta_{1}(z)$ and $\eta_{3}(z)$ will change their sign if the sign of $Q^{*}$ is changed.

Third, we consider the electric potential jump $\Delta \varphi=\varphi_{v}$ $-\varphi_{l}$ across the interface. This spontaneous surface potential jump is attributed to both dipole $\Delta \varphi_{p}$ and quadrupole $\Delta \varphi_{q}$ contributions, i.e., $[16,17,48]$

$$
\begin{gathered}
\Delta \varphi=\Delta \varphi_{p}+\Delta \varphi_{q}, \quad \Delta \varphi_{p}=4 \pi \int_{-\infty}^{+\infty} d z \mu_{0} \eta_{1}(z) \rho(z), \\
\Delta \varphi_{q}=\frac{2 \pi}{3} Q\left(\rho_{l}-\rho_{v}\right) .
\end{gathered}
$$

The quadrupole contribution $\Delta \varphi_{q}$ depends only on $Q$ and the difference in the coexisting liquid and vapor densities, and not on the orientation ordering at the interface. However, the dipole contribution $\Delta \varphi_{p}$ is strongly dependent on the orientation ordering. In Fig. 7(a) we plot the dimensionless potential $\Delta \varphi_{q}^{*} \equiv \Delta \varphi_{q}(d / \epsilon)^{1 / 2}$ on the scaled temperature $\tau$ for given $\left(\mu_{0}^{*}, Q^{*}\right)$. It can be seen that for fixed $\tau$, increasing $\mu_{0}^{*}$ and $Q^{*}$ enlarges $\Delta \varphi_{q}^{*}$, and $\Delta \varphi_{q}^{*}$ is much more sensitive to the change of $Q^{*}$ than $\mu_{0}^{*}$. This is because for fixed $\tau$ the electric potential $\Delta \varphi_{q}^{*}$ is nearly independent of $\mu_{0}^{*}$, but is linearly dependent on $Q^{*}$ as shown by Eq. (42). Near the critical point the density difference $\rho_{l}-\rho_{v}$ obeys the scaling relation Eq. (35). Thus, on the basis of Eq. (42) we find that for fixed $\left(\mu_{0}^{*}, Q^{*}\right)$, potential $\Delta \varphi_{q}^{*}$ satisfies the same mean-field scaling relation as the Eq. (35), i.e.,

$$
\Delta \varphi_{q}^{*} \sim \tau^{1 / 2}
$$

The inset to Fig. 7(a) displays the dipole contribution to the surface potential, $\Delta \varphi_{p}^{*}$, as a function of $\tau$ for the given $\left(\mu_{0}^{*}, Q^{*}\right)$. Note that $\Delta \varphi_{p}$ vanishes for purely dipolar fluids because the $\eta_{1}(z)$ profile is zero [see Eq. (42)]. Moreover, because $\eta_{1}(z)$ is entirely negative across the interface, the
$\Delta \varphi^{*}{ }_{q}$

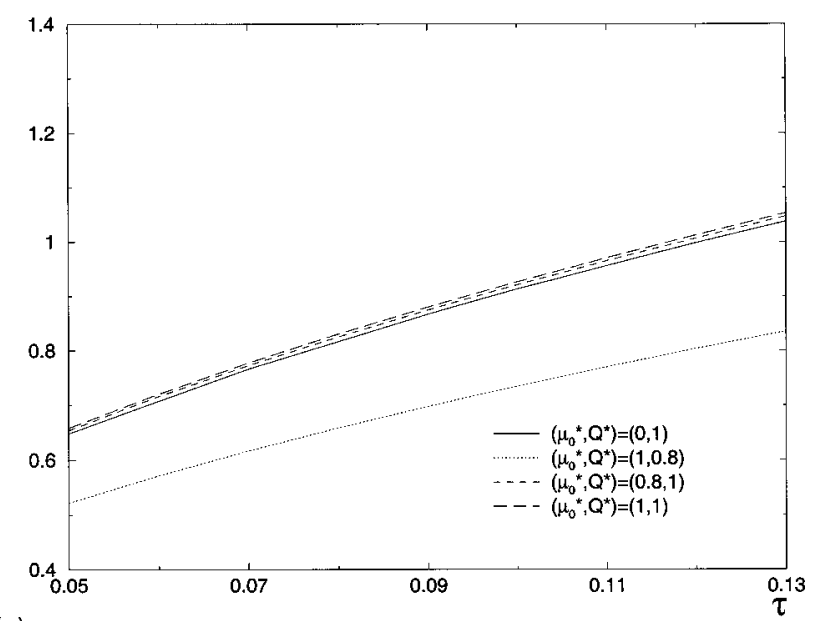

(a)

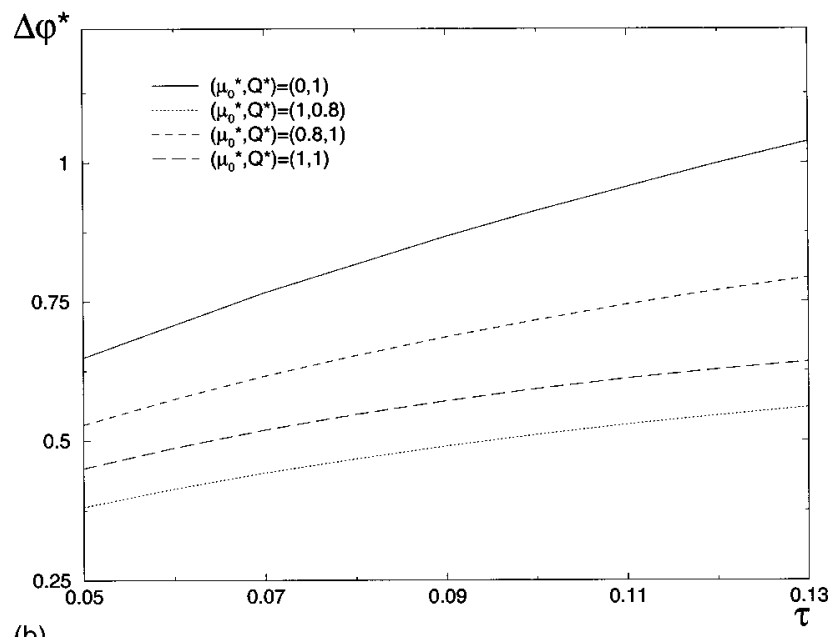

(b)

FIG. 7. The scaled temperature ( $\tau$ ) dependence of (a) the scaled quadrupole surface potential $\Delta \varphi_{q}^{*}$ and (b) the total surface potential $\Delta \varphi^{*}$ for various given $\left(\mu_{0}^{*}, Q^{*}\right)$. The inset to (a) shows the temperature dependence of the dipole surface potential $\Delta \varphi_{p}^{*}$.

potential $\Delta \varphi_{p}^{*}$ is also negative [see Eq. (42)]. In contrast to $\Delta \varphi_{q}^{*}, \Delta \varphi_{p}^{*}$ for given $\tau$ is more sensitive to the change of $\mu_{0}^{*}$ than $Q^{*}$. In Fig. 7(b) we plot the full surface potential $\Delta \varphi^{*}$ versus $\tau$ for the given $\left(\mu_{0}^{*}, Q^{*}\right)$. Because $\left|\Delta \varphi_{p}^{*}\right|$ $<\Delta \varphi_{q}^{*}$, the full surface potential $\Delta \varphi^{*}$ is always positive for the given $\tau$ and $\left(\mu_{0}^{*}, Q^{*}\right)$. Changing the sign of $Q^{*}$ to negative will result in negative $\Delta \varphi_{q}^{*}$ and $\Delta \varphi^{*}$, but $\Delta \varphi_{p}^{*}$ will be positive.

Specifically, the surface potential of water can be calculated by using two popular water models: the ST2 [49] and TIP4P [50]. The reduced dipole and quadrupole moments for the ST2 and TIP4P water models are $\mu_{0}^{*}=1.85$ and $\mu_{0}^{*}$ $=3.68$, and $Q^{*}=0.19$ and $Q^{*}=0.78$, respectively. It can be seen that $\mu_{0}^{*}$ of the TIP4P model is almost twice larger than $\mu_{0}^{*}$ of the ST2 model, and $Q^{*}$ of the TIP4P is almost four times larger than $Q^{*}$ of the ST2. Assuming the present DFT can be extrapolated to treat strongly dipolar-quadrupolar fluid such as ST2 and TIP4P water models, one would 


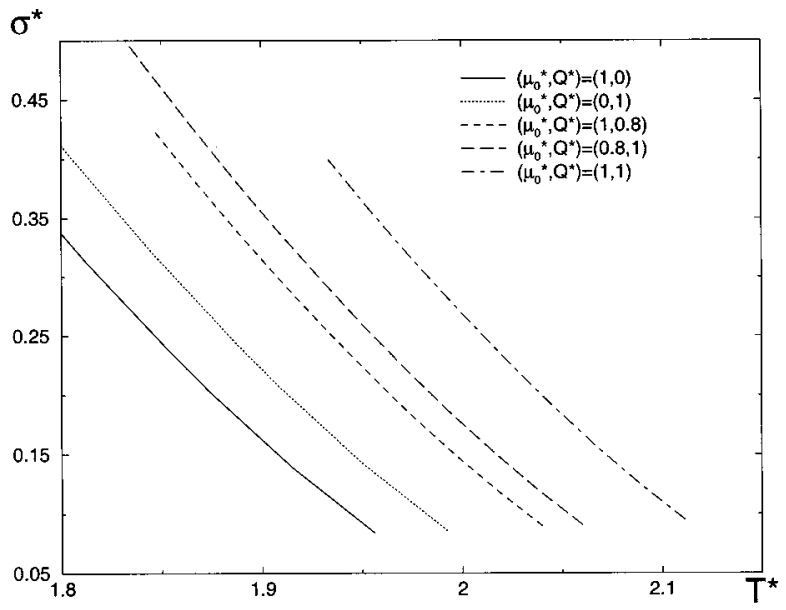

(a)

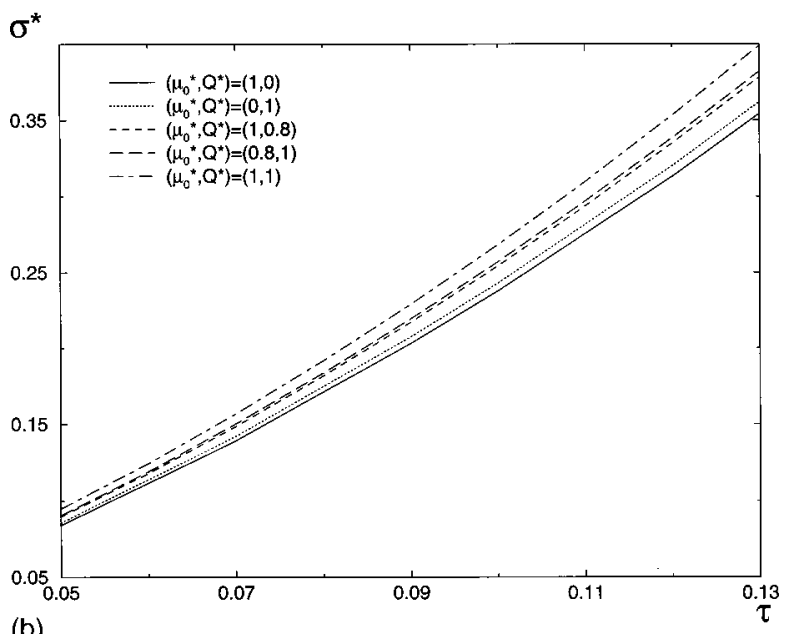

(b)

FIG. 8. (a) Temperature $\left(T^{*}\right)$ and (b) the scaled temperature $(\tau)$ dependence of the scaled surface tension $\sigma^{*} \equiv \sigma d^{2} / \epsilon$ for various given $\left(\mu_{0}^{*}, Q^{*}\right)$.

predict that $\Delta \varphi_{p}(\mathrm{TIP} 4 \mathrm{P}) / \Delta \varphi_{p}(\mathrm{ST} 2)>1, \quad \Delta \varphi(\mathrm{TIP} 4 \mathrm{P}) /$ $\Delta \varphi(\mathrm{ST} 2)>1$, and $\Delta \varphi_{q}(\mathrm{TIP} 4 \mathrm{P}) / \Delta \varphi_{q}(\mathrm{ST} 2)>1$. For both water models, $\Delta \varphi$ and $\Delta \varphi_{p}$ would have opposite signs. This prediction will be in qualitative agreement with previous computer simulation [51] and theory [52] on the spontaneous surface potential of water [53].

Fourth, we consider the dependence of the surface tension on the multipole moment $\left(\mu_{0}^{*}, Q^{*}\right)$. The thermodynamical surface tension $\sigma$ is defined as the excess of the grand potential per unit area

$$
\sigma=\left(\Omega-\Omega^{b u l k}\right) / A \text {. }
$$

In Fig. 8(a) we show the dimensionless surface tension $\sigma^{*}$ $\equiv \sigma\left(d^{2} / \epsilon\right)$ versus $T^{*}$ for various given $\left(\mu_{0}^{*}, Q^{*}\right)$. It can be seen that for fixed $T^{*}$, increasing $\mu_{0}^{*}$ or $Q^{*}$ enlarges the surface tension $\sigma^{*}$. It can be also seen that $\sigma^{*}$ is more sensitive to the change of $Q^{*}$ than $\mu_{0}^{*}$. Haile et al. [1] developed a perturbation method to study the effects of aniso- tropic intermolecular forces on the surface tension. Based on the Fowler model interface (abrupt vapor-liquid transition), they showed that for a given temperature the surface tension of a pure quadrupolar fluid is higher than that of the purely dipolar fluid, assuming the value of $\mu_{0}^{*}$ is the same as $Q^{*}$ (see Fig. 2 in Ref. [1]). Their results are consistent with ours [Fig. 8(a)].

In Fig. 8(b) we plot the surface tension versus the scaled temperature $\tau$ for various given $\left(\mu_{0}^{*}, Q^{*}\right)$. Because these surface tension curves do not collapse onto a single master curve, we conclude that the principle of corresponding states is not satisfied as far as the surface tension of the dipolarquadrupolar fluid is concerned. In contrast, for a purely dipolar fluid, Teixeira and Telo da Gama [2] find that the surface tension $\sigma^{*}$ does obey the principle of corresponding states. Near the critical point the surface tension still satisfies the mean-field scaling relation

$$
\sigma^{*} \sim \tau^{3 / 2}
$$

Finally, we note that the surface tension $\sigma^{*}$ is independent of the sign of $Q^{*}$.

\section{B. Nonzero field}

The electric field effects on the interfacial order-parameter profiles $\eta_{1}(z)$ and $\eta_{2}(z)$ are shown in Fig. 9 for $T^{*}=1.9$ and $\left(\mu_{0}^{*}, Q^{*}\right)=(1,0.8)$. Figure 9 (a) shows that when the applied field is in the direction from the liquid to vapor (referred as the positive field $E^{*}>0$ ), values of $\left|\eta_{1}(z)\right|$ are always less than or equal to those if the direction of the field is reversed (i.e., the negative field $E^{*}<0$ ). Note that this "symmetry breaking" behavior by reversing the field direction never occurs in purely dipolar fluids. In the dipolarquadrupolar fluid, however, the positive field induces a local orientational ordering that offsets the spontaneous ordering (produced in the zero field) at the interface. On the other hand, in the case of negative field the field-induced ordering enhances the spontaneous interfacial ordering. Indeed, as shown in Fig. 9(b), the positive field increases values of $\eta_{2}(z)$ [compared to $\eta_{2}^{(0)}(z)$ ] on the vapor side and decreases $\eta_{2}(z)$ on the liquid side. In contrast, the negative field reduces $\eta_{2}(z)$ on the vapor side and enlarges it on the liquid side. This contrast becomes more pronounced as the magnitude of the field becomes greater.

In Fig. 10, we plot the $\sigma^{*}-T^{*}$ curve for three given values of $E^{*}$. It can be seen that for fixed $T^{*}$ the electric field reduces the surface tension $\sigma^{*}$. However, the reduction in the surface tension is larger for the negative field (for example, when negative charges are put in the liquid) than the positive field (when positive charges are put in the liquid). This result is consistent with the conclusion about the dependence of the surface tension on the external field for a liquid having a negative spontaneous surface potential $\Delta \varphi_{p}$ (see Refs. [18,37]). As an example, for $T^{*}=1.9$ the positive field changes the surface tension by about $0.4 \%$, whereas the negative field changes it by about $1.3 \%$. As a result, the Gibbs-Volmer critical nucleus formed on a positive-charge 


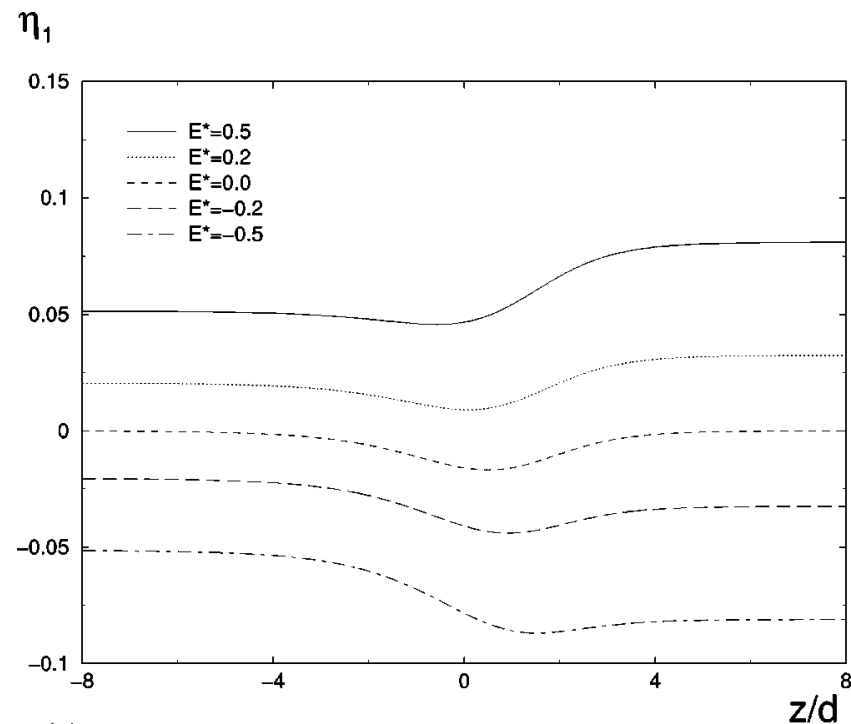

(a)

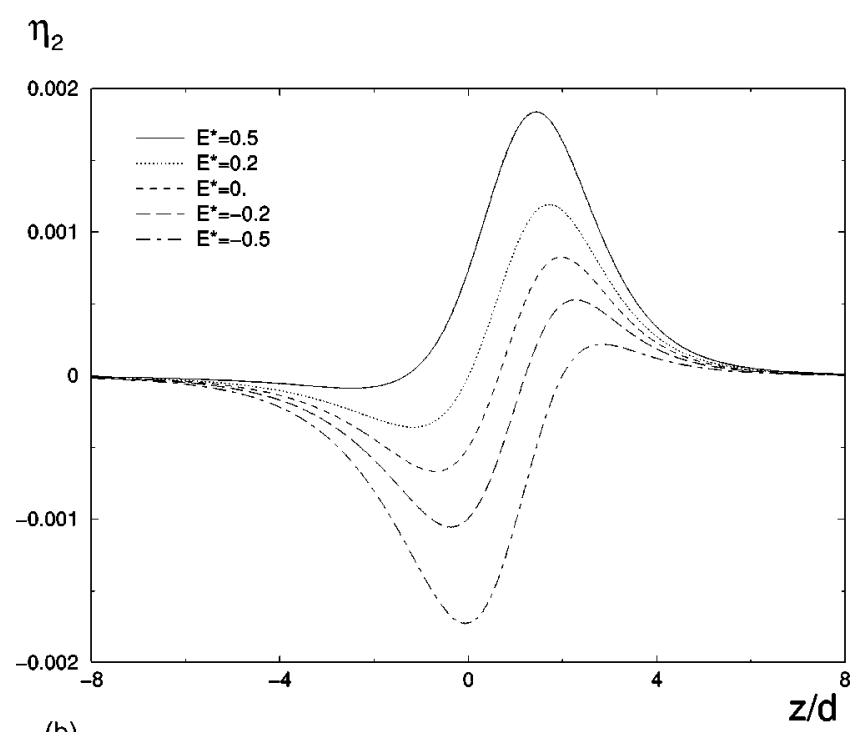

(b)

FIG. 9. The order-parameter profile (a) $\eta_{1}(z)$ and (b) $\eta_{2}(z)$ at the interface for $T^{*}=1.9,\left(\mu_{0}^{*}, Q^{*}\right)=(1,0.8)$, and various given $E^{*}$.

center will be bigger than that formed on a negative-charge center. As the Gibbs-Volmer critical nucleus is produced via the thermal fluctuation, the formation of the critical nucleus on the negative-charge particle will be more probable and the homogeneous nucleation will proceed faster on negativecharge condensation centers assuming their quadrupole moment $Q^{*}$ is positive. If $Q^{*}$ is negative, the reduction in the surface tension will be larger in the positive field, thereby promotes faster nucleation on the positive-charge condensation centers.

\section{CONCLUSIONS}

We have extended the modified mean-field densityfunctional theory [2] to study the bulk and interfacial properties of a dipolar-quadrupolar fluid in a slab-shaped system. We have explored the effects of a uniform electric field on

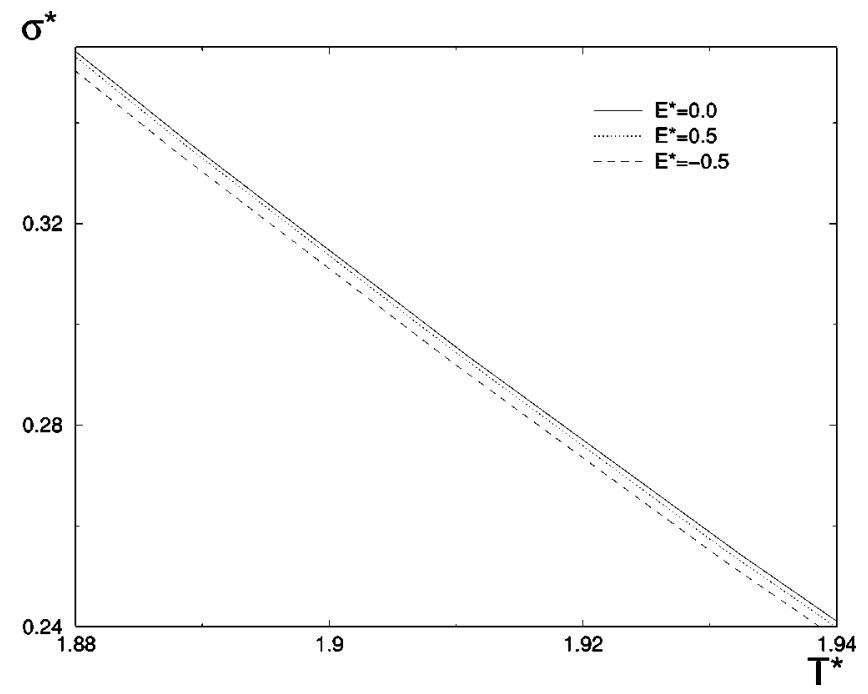

FIG. 10. Temperature $\left(T^{*}\right)$ dependence of the surface tension $\sigma^{*}$ for $\left(\mu_{0}^{*}, Q^{*}\right)=(1,0.8)$ in the zero field and in a positive and a negative field, respectively, with the same magnitude $E^{*}=0.5$.

the bulk and interfacial properties of the fluid. Specifically, we assumed the field is normal to the surfaces of the slab. We find that increasing the multipole (dipole or quadrupole) moment will increase the critical temperature and broaden the vapor-liquid coexistence curve. The enhancement of critical temperature and broadening of the coexistence curve is more sensitive to the change in quadrupole moment $Q^{*}$ than the dipole moment $\mu_{0}^{*}$, as also shown in previous work $[11,14]$. When a uniform electric field is applied perpendicular to the surfaces of the slab, we find that the field reduces the critical temperature and narrows the coexistence curve. We also find that in the electric field and near the critical temperature the difference in the coexistence density $\rho_{l}-\rho_{v}$ and order parameter $\eta_{1 v}-\eta_{1 l}$ both scales as $\tau^{1 / 2} \quad[\tau \equiv 1$ $\left.-T / T_{c}\left(\mu_{0}, Q, E\right)\right]$. For small values of $\mu_{0}^{*}$ and $Q^{*}$, the coexistence densities satisfy the law of the corresponding states. The sign of quadrupole moment $Q^{*}$ and field direction have no effect on the coexistence curve but the order parameter $\eta_{1}$ is dependent on the field direction.

For the planar vapor-liquid interface, even in the zero field the dipolar-quadrupolar molecules have preferred orientation at the interface. The fact that the order parameter $\eta_{2}(z)=\left\langle P_{2}\right\rangle$ is negative on the liquid side and positive on the vapor side indicates that molecules tend to lie in parallel with the interface on the liquid side but tend to lie in perpendicular to the interface on the vapor side. Moreover, the fact that the order parameter $\eta_{1}(z)=\left\langle P_{1}\right\rangle$ is negative across the interface indicates that the molecules prefer to point the dipole towards the liquid. The interfacial ordering characterized by $\left\langle P_{1}\right\rangle$ is due to the coupling effect of molecular dipoles and quadrupoles; the ordering is nonexistent for purely dipolar or quadrupolar fluids. In contrast, the interfacial ordering characterized by $\left\langle P_{2}\right\rangle$ exists even for purely dipolar and purely quadrupolar fluids. Near the critical point, the degree of the orientation order described by parameter $D_{\eta_{1}}$ approaches to zero as $\tau$, and the degree of the orientation order described by the parameter $D_{\eta_{2}}$ as $\tau^{3 / 2}$. 
At a fixed temperature we find that increasing the multipolar (dipolar or quadrupolar) moment will lessen the width of interface $W$, as well as the widths of the order-parameter profiles $W_{\eta_{1}}$ and $W_{\eta_{2}}$; it will result in a sharp change in the density profile, increase the degrees of the molecular orders $D_{\eta_{1}}$ and $D_{\eta_{2}}$, enhance the surface potential due to quadrupoles $\Delta \varphi_{q}^{*}$ and the absolute value of surface potential due to dipoles $\left|\Delta \varphi_{p}^{*}\right|$, and the surface tension $\sigma^{*}$. Near the critical point, we find that $\Delta \varphi_{q}^{*}$ scales as $\tau^{1 / 2}$ and $\sigma^{*}$ as $\tau^{3 / 2}$. These mean-field scaling relations hold better for small values of $\mu_{0}^{*}$ and $Q^{*}$. We also find that many interfacial characteristics $\left(W, D_{\eta_{1}}, D_{\eta_{2}}, W_{\eta_{1}}, W_{\eta_{2}}, \Delta \varphi^{*}\right.$, and $\left.\sigma^{*}\right)$ are more sensitive to the change of the dimensionless value $Q^{*}$ than $\mu_{0}^{*}$. Moreover, changing the sign of $Q^{*}$ has no effects on the order-parameter profile $\eta_{2}(z)$ and the surface tension $\sigma^{*}$, but it does affect the order-parameter profile $\eta_{1}(z)$ and the spontaneous surface potential $\Delta \varphi^{*}$.

Finally, we find that at a fixed temperature the applied electric field reduces the surface tension $\sigma^{*}$. For a given positive $Q^{*}$ the reduction in the surface tension due to the field is greater if the field is in the direction from vapor to liquid, compared to the field in the opposite direction. This surface tension dependence on the field direction does not occur for purely dipolar fluids. We conclude that this apparent "symmetry breaking" by reversing the field direction suggests a molecular mechanism, without invoking explicit anisotropic molecular structure, to explain the sign preference in droplet formation on charged condensation center. This can be understood as follows: when a negative charge (anion) is introduced into a liquid droplet, the anion yields a negative field, that is, the direction of the field is from vapor to the liquid droplet. Obviously, the cation counterpart will yield an opposite positive field from the droplet to vapor. Since the anion yields a smaller surface tension than the cation counterpart (assuming $Q^{*}>0$ ), the classical nucleation theory [37] should predict that the anion will lead to a smaller barrier to the droplet formation $\left(\Delta G^{*}\right)$ than the cation counterpart. Because the rate of heterogeneous nucleation is proportional to $e^{-\Delta G^{*} / k_{B} T}$, the ion-induced critical nuclei are more likely to form on negative charged particles. Consequently, the fluid will have a negative sign preference in the droplet formation on charged condensation center. On the other hand, if $Q^{*}<0$, the dipolar-quadrupolar fluid will have a positive sign preference in droplet formation.

\section{ACKNOWLEDGMENTS}

This work was supported by the National Science Foundation and in part by the Research Computing Facility of the University of Nebraska-Lincoln.

\section{APPENDIX A}

In this section we derive the effective potential $\phi_{\text {eff }}$ arising in Eqs. (15) and (16). Expressing vector $\vec{r}_{12}$ in the cylindrical coordinates $\left[\vec{r}_{12}=\left(R_{12}, z_{12}, \varphi_{12}\right)\right]$, from Eqs. (13) and (14) we obtain Eq. (15), in which $\phi_{e f f}$ is given by [2]

$$
\begin{aligned}
\phi_{e f f}\left(z_{12}, \omega_{1}, \omega_{2}\right)= & \int_{0}^{2 \pi} d \varphi_{12} \int_{0}^{+\infty} R_{12} d R_{12} \\
& \times\left\{u_{p e r}\left(R_{12}, z_{12}, \varphi_{12}, \omega_{1}, \omega_{2}\right)\right. \\
& \left.-\frac{\beta}{2} u_{p e r}^{2}\left(R_{12}, z_{12}, \varphi_{12}, \omega_{1}, \omega_{2}\right)\right\} .
\end{aligned}
$$

Using the expression for $u_{p e r}$ [Eq. (2)], Eq. (A1) can be rewritten as

$$
\begin{aligned}
\phi_{e f f}\left(z_{12}, \omega_{1}, \omega_{2}\right)= & \overline{u_{00}}+\overline{u_{d q}}+\overline{u_{q d}}+\overline{u_{d d}}+\overline{u_{q q}}-\frac{\beta}{2}\left[\overline{u_{00}^{2}}+\overline{u_{d d}^{2}}\right. \\
& +2 \overline{u_{00} u_{d d}}+2 \overline{u_{d d} u_{q q}}+2 \overline{u_{00} u_{q q}}+\overline{u_{q q}^{2}} \\
& +\overline{\left(u_{d q}+u_{q d}\right)^{2}}+2 \overline{u_{00}\left(u_{d q}+u_{q d}\right)} \\
& \left.+2 \overline{u_{d d}\left(u_{d q}+u_{q d}\right)}+2 \overline{u_{q q}\left(u_{d q}+u_{q d}\right)}\right]
\end{aligned}
$$

where the bar notation

$$
\begin{aligned}
\bar{v}\left(z_{12}, \omega_{1}, \omega_{2}\right) \equiv & \int_{0}^{2 \pi} d \varphi_{12} \int_{0}^{+\infty} d R_{12} R_{12} \\
& \times v\left(R_{12}, z_{12}, \varphi_{12}, \omega_{1}, \omega_{2}\right) H\left(r_{12}-d\right) .
\end{aligned}
$$

Some terms in Eq. (A2) have been derived before (for purely dipolar and quadrupolar fluids) and can be found in Refs. $[2,46]$ :

$$
\begin{gathered}
\overline{u_{00}}=A, \quad \overline{u_{d d}}=B P_{1} P_{1}^{\prime}, \quad \overline{u_{q q}}=\varphi_{q q} P_{2} P_{2}^{\prime}, \\
\overline{u_{00}^{2}}=C, \quad \overline{u_{00} u_{d d}}=(H / 2) P_{2} P_{2}^{\prime}, \\
\overline{u_{d d}^{2}}=\left(\frac{16}{9} D+\frac{16}{3} E\right)+\left(\frac{4}{9} D+8 E-\frac{8}{3} F+G\right) P_{2} P_{2}^{\prime} \\
+\left(\frac{8}{9} D-\frac{8}{3} E+\frac{1}{3} F\right)\left(P_{2}+P_{2}^{\prime}\right), \\
\overline{u_{00} u_{q q}}=\chi_{00, q q}^{22} P_{2} P_{2}^{\prime}, \\
\overline{u_{q q}^{2}}\left(z, \omega_{1}, \omega_{2}\right)= \\
\chi_{q q, q q}^{00}+\chi_{q q, q q}^{22} P_{2} P_{2}^{\prime}+\chi_{q q, q q}^{44} P_{4} P_{4}^{\prime} \\
+\chi_{q q, q q}^{02}\left(P_{2}+P_{2}^{\prime}\right)+\chi_{q q, q q}^{04}\left(P_{4}+P_{4}^{\prime}\right) \\
+\chi_{q q, q q}^{24}\left(P_{2} P_{4}^{\prime}+P_{2}^{\prime} P_{4}\right),
\end{gathered}
$$

where the expressions for $A, C, B, H$ ( $B$ and $H$ are proportional to $\left.\mu_{0}^{2}\right), D, E, F, G(D, E, F$, and $G$ are proportional to $\left.\mu_{0}^{4}\right), \varphi_{q q}$ (proportional to $Q^{2}$ ) are given by Eq. (A14) in Ref. [2]; the expressions for $\chi_{00, q q}^{22}$ (proportional to $Q^{2}$ ) and $\chi_{q q, q q}^{00}, \chi_{q q, q q}^{22}, \chi_{q q, q q}^{44}, \chi_{q q, q q}^{02}, \chi_{q q, q q}^{04} \chi_{q q, q q}^{24}$ (these six functions are proportional to $Q^{4}$ ) are given by Eqs. (A10) and (A14) in Ref. [46] Note that in Eq. (A4) those terms that 
involve $\cos \left[n\left(\varphi_{1}-\varphi_{2}\right)\right]$ are not considered here because the orientation distribution $\hat{f}$ is independent of $\varphi$ and thus the integration over $\varphi$ for those terms vanishes.

Now we present the results for the rest terms in Eq. (A2). These terms are due to coupling effects of dipoles and quadrupoles. To this end, we write the interaction energy $u_{l_{1} l_{2}}$ between the multipoles of order $l_{1}$ and $l_{2}$ (for linear molecules) as a sum of spherical harmonics $Y_{l m}$ [44],

$$
\begin{aligned}
u_{l_{1} l_{2}}= & \frac{A_{l_{1} l_{2}}}{r_{12}^{l_{3}+1}} Q_{l_{1}} Q_{l_{2}} \sum_{m_{1}, m_{2}, m_{3}} C\left(l_{1} l_{2} l_{3} ; m_{1}, m_{2}, m_{3}\right) \\
& \times Y_{l_{1} m_{1}}\left(\omega_{1}\right) Y_{l_{2} m_{2}}\left(\omega_{2}\right) Y_{l_{3} m_{3}}^{*}\left(\omega_{12}\right),
\end{aligned}
$$

where $\left|m_{1}\right| \leqslant l_{1},\left|m_{2}\right| \leqslant l_{2}, m_{3}=m_{1}+m_{2}, l_{3}=l_{1}+l_{2}, Q_{1}$ $=\mu_{0}, \quad Q_{2}=Q, \quad C\left(l_{1} l_{2} l_{3} ; m_{1}, m_{2}, m_{3}\right) \quad$ are the ClebschGordan coefficients in the Rose convention [54], and $\omega_{12}$ denotes the orientation of the intermolecular axis in the space-fixed coordinate system. Coefficients $A_{l_{1} l_{2}}$ are given by [44]

$$
A_{l_{1} l_{2}}=\frac{(-1)^{l_{2}}}{\left(2 l_{3}+1\right)}\left[\frac{(4 \pi)^{3}\left(2 l_{3}+1\right) !}{\left(2 l_{1}+1\right) !\left(2 l_{2}+1\right) !}\right]^{1 / 2} .
$$

We now consider the terms involving multipoles to the second order. Substituting Eq. (A5) with $l_{1}=1, l_{2}=2$ into Eq. (A3), we obtain

$$
\begin{aligned}
\overline{u_{p q}}= & \frac{(4 \pi)^{3 / 2}}{7^{1 / 2}} \mu_{0} Q \sum_{\substack{m_{3}=-3 \\
m_{1}+m_{2}=m_{3}}}^{3} C\left(123 ; m_{1}, m_{2}, m_{3}\right) \\
& \times Y_{1 m_{1}}\left(\omega_{1}\right) Y_{2 m_{2}}\left(\omega_{2}\right) \int_{0}^{+\infty} \frac{R_{12} d R_{12}}{r_{12}^{4}} \\
& \times H\left(r_{12}-d\right) \int_{0}^{2 \pi} Y_{3 m_{3}}^{*}\left(\omega_{12}\right) d \varphi_{12} .
\end{aligned}
$$

Using the relation between the spherical harmonics and associated Legendre polynomials

$$
\begin{aligned}
Y_{l m}(\theta, \varphi)= & (-1)^{m}\left(\frac{(2 l+1)}{4 \pi}\right)^{1 / 2}\left(\frac{(l-m) !}{(l+m) !}\right)^{1 / 2} \\
& \times P_{l}^{m}(\cos \theta) e^{i m \varphi},
\end{aligned}
$$

the relation

$$
Y_{l,-m}(\omega)=(-1)^{m} Y_{l m}^{*}(\omega)
$$

and

$$
P_{l}^{-m}(x)=(-1)^{m} \frac{(l-m) !}{(l+m) !} P_{l}^{m}(x),
$$

and the orthogonal relation $\int_{0}^{2 \pi} e^{i\left(m-m^{\prime}\right) \varphi} d \varphi=2 \pi \delta_{m m^{\prime}}$, Eq. (A7) can be written as

$$
\begin{aligned}
\overline{u_{p q}}\left(z_{12}, \omega_{1}, \omega_{2}\right)= & 8 \pi^{2} \mu_{0} Q \sum_{m_{1}+m_{2}=0} C\left(123 ; m_{1}, m_{2}, 0\right) \\
& \times Y_{1 m_{1}}\left(\omega_{1}\right) Y_{2 m_{2}}\left(\omega_{2}\right) \int_{0}^{+\infty} \frac{R_{12} d R_{12}}{r_{12}^{4}} \\
& \times H\left(r_{12}-d\right) P_{3}\left(\cos \theta_{12}\right) .
\end{aligned}
$$

Since $P_{3}\left(\cos \theta_{12}\right)$ in the above integrand is a polynomial of $\cos \theta_{12}=z_{12} / r_{12}$ of order 3 , the integral in Eq. (A8) can be reduced to a sum of the integral $\int_{0}^{+\infty} R_{12} d R_{12} / r_{12}^{n} H\left(r_{12}-d\right)$, where $n$ is an integer greater than 2. With $r_{12}=\sqrt{R_{12}^{2}+z_{12}^{2}}$ we obtain

$$
\int_{0}^{+\infty} \frac{R_{12} d R_{12}}{r_{12}^{n}} H\left(r_{12}-d\right)=\frac{1}{(n-2)} \begin{cases}\frac{1}{d^{n-2}}, & \left|z_{12}\right| \leqslant d \\ \frac{1}{\left|z_{12}\right|^{n-2}}, & \left|z_{12}\right|>d .\end{cases}
$$

Substituting Eq. (A9) into Eq. (A8) gives

$$
\overline{u_{d q}}=\chi_{d q}^{12} P_{1} P_{2}^{\prime},
$$

where

$$
\chi_{d q}^{12}=\frac{\pi \mu_{0} Q}{d^{2}}\left\{\begin{array}{l}
3\left(\frac{z^{3}}{d^{3}}-\frac{z}{d}\right), \quad|z| \leqslant d \\
0, \quad|z|>d .
\end{array}\right.
$$

Equations (A5) and (A6), and the relation $C\left(213 ; m_{2} m_{1} m\right)$ $=C\left(123 ; m_{1} m_{2} m\right)$ [44] together result in the relation $\bar{u}_{12}\left(z_{12}, \omega_{1}, \omega_{2}\right)=-\bar{u}_{21}\left(z_{12}, \omega_{2}, \omega_{1}\right)$. Thus,

$$
\overline{u_{q d}}=-\chi_{d q}^{12} P_{1}^{\prime} P_{2} \text {. }
$$

A derivation of $\overline{u_{00}\left(u_{d q}+u_{q d}\right)}$ is straightforward because it has the same angular dependence as $\overline{\left(u_{d q}+u_{q d}\right)}$, that is

$$
\overline{u_{00}\left(u_{d q}+u_{q d}\right)}=\chi_{00, d q}^{12}\left(P_{1} P_{2}^{\prime}-P_{1}^{\prime} P_{2}\right)
$$

where

$$
\chi_{00, d q}^{12}=-\epsilon \frac{\pi \mu_{0}^{2} Q^{2}}{d^{2}} 72 \sqrt{\frac{19}{7}}\left\{\begin{array}{l}
\frac{5}{11} \frac{z^{3}}{d^{3}}-\frac{1}{3} \frac{z}{d}, \quad|z| \leqslant 1 \\
\frac{4}{33} \frac{z d^{8}}{\left|z^{9}\right|}, \quad|z|>d .
\end{array}\right.
$$

Derivation of other terms, $\overline{u_{d d} u_{q q}}, \overline{\left(u_{d q}+u_{q d}\right)^{2}}$, $\overline{u_{d d}\left(u_{d q}+u_{q d}\right)}$, and $\overline{u_{q q}\left(u_{d q}+u_{q d}\right)}$ requires the expression

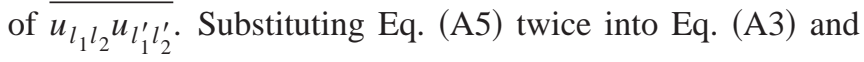
taking the integration over the angle $\varphi_{12}$ yields 


$$
\begin{aligned}
\overline{u_{l_{1} l_{2}} u_{l_{1}^{\prime} l_{2}^{\prime}}}= & A_{l_{1} l_{2}} A_{l_{1}^{\prime} l_{2}^{\prime}} Q_{l_{1}} Q_{l_{2}} Q_{l_{1}^{\prime}} Q_{l_{2}^{\prime}} \frac{\left[\left(2 l_{3}+1\right)\left(2 l_{3}^{\prime}+1\right)\right]^{1 / 2}}{2} \sum_{m_{3}=-l_{3}^{\prime \prime}}^{l_{3}^{\prime \prime}}\left[\frac{\left(l_{3}-m_{3}\right) !\left(l_{3}^{\prime}-m_{3}\right) !}{\left(l_{3}+m_{3}\right) !\left(l_{3}^{\prime}+m_{3}\right) !}\right]^{1 / 2} \\
& \times C\left(l_{1} l_{2} l_{3} ; m_{1}, m_{2}, m_{3}\right) C\left(l_{1}^{\prime} l_{2}^{\prime} l_{3}^{\prime} ; m_{1}^{\prime}, m_{2}^{\prime},-m_{3}\right) Y_{l_{1} m_{1}}\left(\omega_{1}\right) Y_{l_{1}^{\prime} m_{1}}^{*}\left(\omega_{1}\right) Y_{l_{2} m_{2}}\left(\omega_{2}\right) Y_{l_{2}^{\prime} m_{2}}^{*}\left(\omega_{2}\right) \\
& \times \int_{0}^{+\infty} \frac{R_{12} d R_{12}}{r_{12}^{l_{3}+l_{3}^{\prime}+2} H\left(r_{12}-d\right) P_{l_{3}}^{m_{3}}\left(\cos \theta_{12}\right) P_{l_{3}^{\prime}}^{-m_{3}}\left(\cos \theta_{12}\right)}
\end{aligned}
$$

where $m_{1}+m_{2}=m_{3}, \quad m_{1}^{\prime}+m_{2}^{\prime}=-m_{3}$, and $l_{3}^{\prime \prime}=\min \left(l_{3}, l_{3}^{\prime}\right)$. $P_{l_{3}}^{m_{3}}\left(\cos \theta_{12}\right) P_{l_{3}^{\prime}}^{-m_{3}}\left(\cos \theta_{12}\right)$ are polynomials of $\cos \theta_{12}$ $=z_{12} / r_{12}$ with the highest order $l_{3}+l_{3}^{\prime}$. With the necessary $l_{1}, l_{2}, l_{1}^{\prime}, l_{2}^{\prime}$, Eq. (A9) and some relevant relations for the Clebsch-Gordan coefficients and spherical harmonics [44], all the required $\overline{u_{l_{1} l_{2}} u_{l_{1}^{\prime} l_{2}^{\prime}}}$ can be obtained after some lengthy algebraic manipulation. The results are detailed below:

$\overline{u_{d d} u_{q q}}=\chi_{d d, q q}^{11} P_{1} P_{1}^{\prime}+\chi_{d d, q q}^{33} P_{3} P_{3}^{\prime}+\chi_{d d, q q}^{13}\left(P_{1} P_{3}^{\prime}+P_{1}^{\prime} P_{3}\right)$,

where

$$
\begin{aligned}
& \chi_{d d, q q}^{11}=-\frac{\pi \mu_{0}^{2} Q^{2}}{d^{6}} \frac{3}{5}\left\{\begin{array}{l}
\frac{9}{5} \frac{z^{2}}{d^{2}}-\frac{4}{5}, \quad|z| \leqslant d \\
\frac{d^{6}}{z^{6}}, \quad|z|>d,
\end{array}\right. \\
& \chi_{d d, q q}^{33}=-\frac{\pi \mu_{0}^{2} Q^{2}}{d^{6}} \frac{3}{80} \\
& \times\left\{\begin{array}{l}
350 \frac{z^{6}}{d^{6}}-580 \frac{z^{4}}{d^{4}}+\frac{1239}{5} \frac{z^{2}}{d^{2}}-\frac{84}{5}, \quad|z| \leqslant d \\
\frac{d^{6}}{z^{6}}, \quad|z|>d,
\end{array}\right. \\
& \chi_{d d, q q}^{13}=-\frac{\pi \mu_{0}^{2} Q^{2}}{d^{6}} \frac{3}{20}\left\{\begin{array}{l}
10 \frac{z^{4}}{d^{4}}-\frac{51}{5} \frac{z^{2}}{d^{2}}+\frac{6}{5}, \quad|z| \leqslant d \\
\frac{d^{6}}{z^{6}}, \quad|z|>d ;
\end{array}\right. \\
& \overline{\left(u_{d q}+u_{q d}\right)^{2}}=\chi_{d q, d q}^{00}+\chi_{d q, d q}^{11} P_{1} P_{1}^{\prime}+\chi_{d q, d q}^{22} P_{2} P_{2}^{\prime} \\
& +\chi_{d q, d q}^{33} P_{3} P_{3}^{\prime}+\chi_{d q, d q}^{02}\left(P_{2}+P_{2}^{\prime}\right) \\
& +\chi_{d q, d q}^{04}\left(P_{4}+P_{4}^{\prime}\right)+\chi_{d q, d q}^{13}\left(P_{1} P_{3}^{\prime}+P_{1}^{\prime} P_{3}\right) \\
& +\chi_{d q, d q}^{24}\left(P_{2} P_{4}^{\prime}+P_{2}^{\prime} P_{4}\right) \text {, }
\end{aligned}
$$

where

$$
\chi_{d q, d q}^{00}=\frac{\pi \mu_{0}^{2} Q^{2}}{d^{6}} \frac{2}{3} \begin{cases}1, & |z| \leqslant d \\ \frac{d^{6}}{z^{6}}, & |z|>d,\end{cases}
$$

$$
\chi_{d q, d q}^{11}=\frac{\pi \mu_{0}^{2} Q^{2}}{d^{6}} \frac{3}{50}\left\{\begin{array}{l}
50 \frac{z^{6}}{d^{6}}-84 \frac{z^{4}}{d^{4}}+45 \frac{z^{2}}{d^{2}}+4, \quad|z| \leqslant d \\
15 \frac{d^{6}}{z^{6}}, \quad|z|>d,
\end{array}\right.
$$

$$
\begin{aligned}
& \chi_{d q, d q}^{33}=\frac{\pi \mu_{0}^{2} Q^{2}}{d^{6}} \frac{3}{100} \\
& \times\left\{\begin{array}{l}
375 \frac{z^{6}}{d^{6}}-600 \frac{z^{4}}{d^{4}}+\frac{513}{2} \frac{z^{2}}{d^{2}}-4, \quad|z| \leqslant d \\
\frac{55}{2} \frac{d^{6}}{z^{6}}, \quad|z|>d,
\end{array}\right.
\end{aligned}
$$$$
\chi_{d q, d q}^{02}=\frac{\pi \mu_{0}^{2} Q^{2}}{d^{6}} \frac{17}{42}\left\{\begin{array}{l}
\frac{9}{5} \frac{z^{2}}{d^{2}}-\frac{4}{5}, \quad|z| \leqslant d \\
\frac{d^{6}}{z^{6}}, \quad|z|>d
\end{array}\right.
$$

$$
\chi_{d q, d q}^{04}=\frac{\pi \mu_{0}^{2} Q^{2}}{d^{6}} \frac{1}{6}\left\{\begin{array}{l}
\frac{9}{2} \frac{z^{4}}{d^{4}}-\frac{135}{28} \frac{z^{2}}{d^{2}}+\frac{9}{14}, \quad|z| \leqslant d \\
\frac{9}{28} \frac{d^{6}}{z^{6}}, \quad|z|>d,
\end{array}\right.
$$




$$
\begin{aligned}
\chi_{d q, d q}^{13}= & \frac{\pi \mu_{0}^{2} Q^{2}}{d^{6}} \frac{3}{50}\left\{\begin{array}{l}
\frac{25}{2} \frac{z^{6}}{d^{6}}+9 \frac{z^{4}}{d^{4}}-\frac{75}{4} \frac{z^{2}}{d^{2}}+1, \quad|z| \leqslant d \\
\frac{15}{4} \frac{d^{6}}{z^{6}}, \quad|z|>d,
\end{array}\right. \\
\chi_{d q, d q}^{24}= & \frac{\pi \mu_{0}^{2} Q^{2}}{d^{6}} \frac{1}{6} \\
& \times\left\{\begin{array}{l}
\frac{225}{4} \frac{z^{6}}{d^{6}}-\frac{1305}{14} \frac{z^{4}}{d^{4}}+\frac{1593}{40} \frac{z^{2}}{d^{2}}-\frac{27}{10}, \quad|z| \leqslant d \\
\frac{9}{56} \frac{d^{6}}{z^{6}}, \quad|z|>d ;
\end{array}\right.
\end{aligned}
$$$$
\overline{u_{q q}\left(u_{d q}+u_{q d}\right)}=\chi_{d q, q q}^{01}\left(P_{1}^{\prime}-P_{1}\right)+\chi_{d q, q q}^{03}\left(P_{3}^{\prime}-P_{3}\right)
$$$$
+\chi_{d q, q q}^{12}\left(P_{1} P_{2}^{\prime}-P_{1}^{\prime} P_{2}\right)+\chi_{d q, q q}^{14}\left(P_{1} P_{4}^{\prime}\right.
$$$$
\left.-P_{1}^{\prime} P_{4}\right)+\chi_{d q, q q}^{23}\left(P_{2} P_{3}^{\prime}-P_{2}^{\prime} P_{3}\right)
$$$$
+\chi_{d q, q q}^{34}\left(P_{3} P_{4}^{\prime}-P_{3}^{\prime} P_{4}\right) \text {, }
$$

where

$$
\begin{aligned}
\overline{u_{d d}\left(u_{d q}+u_{q d}\right)}= & \chi_{d d, d q}^{01}\left(P_{1}^{\prime}-P_{1}\right)+\chi_{d d, d q}^{03}\left(P_{3}^{\prime}-P_{3}\right) \\
& +\chi_{d d, d q}^{12}\left(P_{2}^{\prime} P_{1}-P_{1}^{\prime} P_{2}\right)+\chi_{d d, d q}^{23} \\
& \times\left(P_{2} P_{3}^{\prime}-P_{2}^{\prime} P_{3}\right),
\end{aligned}
$$

where

$$
\begin{aligned}
& \chi_{d d, d q}^{01}=-\frac{\pi \mu_{0}^{3} Q}{d^{5}} \frac{2}{5} \frac{12}{5} \begin{cases}\frac{z}{d}, & |z| \leqslant d \\
\frac{d^{5}}{z^{5}}, & |z|>d,\end{cases} \\
& \chi_{d d, d q}^{03}=-\frac{\pi \mu_{0}^{3} Q}{d^{5}} \frac{1}{10}\left\{\begin{array}{l}
5 \frac{z^{3}}{d^{3}}-4 \frac{z}{d}, \quad|z| \leqslant d \\
\frac{d^{5}}{z^{5}}, \quad|z|>d,
\end{array}\right. \\
& \chi_{d d, d q}^{12}=\frac{\pi \mu_{0}^{3} Q}{d^{5}} \frac{1}{10}\left\{\begin{array}{l}
6 \frac{z^{3}}{d^{3}}-4 \frac{z}{d}, \quad|z| \leqslant d \\
2 \frac{d^{5}}{z^{5}}, \quad|z|>d
\end{array}\right. \\
& \chi_{d d, d q}^{23}=-\frac{\pi \mu_{0}^{3} Q}{d^{5}} \frac{1}{10}\left\{\begin{array}{l}
90 \frac{z^{5}}{d^{5}}-127 \frac{z^{3}}{d^{3}}+38 \frac{z}{d}, \quad|z| \leqslant d \\
\frac{d^{5}}{z^{5}}, \quad|z|>d ;
\end{array}\right.
\end{aligned}
$$

$$
\begin{aligned}
\chi_{d q, q q}^{01}= & -\frac{\pi \mu_{0} Q^{3}}{d^{7}} \frac{3}{10} \\
& \times\left\{\begin{array}{l}
5 \frac{z^{7}}{d^{7}}-\frac{19}{2} \frac{z^{5}}{d^{5}}+5 \frac{z^{3}}{d^{3}}+\frac{5}{4} \frac{z}{d}, \quad|z| \leqslant d \\
\frac{7}{4} \frac{d^{7}}{z^{7}}, \quad|z|>d,
\end{array}\right.
\end{aligned}
$$

$$
\begin{aligned}
\chi_{d q, q q}^{03}= & -\frac{\pi \mu_{0} Q^{3}}{d^{7}} \frac{3}{10} \\
& \times\left\{\begin{array}{l}
-5 \frac{z^{7}}{d^{7}}+\frac{19}{2} \frac{z^{5}}{d^{5}}-3 \frac{z^{3}}{d^{3}}-\frac{3}{4} \frac{z}{d}, \quad|z| \leqslant d \\
\frac{3}{4} \frac{d^{7}}{z^{7}}, \quad|z|>d,
\end{array}\right.
\end{aligned}
$$$$
\chi_{d q, q q}^{12}=\frac{\pi \mu_{0} Q^{3}}{d^{7}} \frac{3}{35}
$$$$
\times\left\{\begin{array}{l}
25 \frac{z^{7}}{d^{7}}-\frac{95}{2} \frac{z^{5}}{d^{5}}+37 \frac{z^{3}}{d^{3}}-\frac{43}{4} \frac{z}{d}, \quad|z| \leqslant d \\
\frac{15}{4} \frac{d^{7}}{z^{7}}, \quad|z|>d,
\end{array}\right.
$$

$$
\chi_{d q, q q}^{14}=\frac{\pi \mu_{0} Q^{3}}{d^{7}} \frac{9}{280}
$$

$$
\times\left\{\begin{array}{l}
125 \frac{z^{7}}{d^{7}}-\frac{335}{2} \frac{z^{5}}{d^{5}}+37 \frac{z^{3}}{d^{3}}+\frac{9}{4} \frac{z}{d}, \quad|z| \leqslant d \\
-\frac{13}{4} \frac{d^{7}}{z^{7}}, \quad|z|>d,
\end{array}\right.
$$

$$
\begin{aligned}
\chi_{d q, q q}^{23}= & -\frac{\pi \mu_{0} Q^{3}}{d^{7}} \frac{3}{35} \\
& \times\left\{\begin{array}{l}
-25 \frac{z^{7}}{d^{7}}+85 \frac{z^{5}}{d^{5}}-77 \frac{z^{3}}{d^{3}}+\frac{39}{2} \frac{z}{d}, \quad|z| \leqslant d \\
\frac{5}{2} \frac{d^{7}}{z^{7}}, \quad|z|>d,
\end{array}\right.
\end{aligned}
$$

and 


$$
\begin{aligned}
\chi_{d q, q q}^{34}= & \frac{\pi \mu_{0} Q^{3}}{d^{7}} \frac{18}{560} \\
& \times\left\{\begin{array}{l}
755 \frac{z^{7}}{d^{7}}-\frac{2859}{2} \frac{z^{5}}{d^{5}}+791 \frac{z^{3}}{d^{3}}-\frac{439}{4} \frac{z}{d}, \quad|z| \leqslant d \\
\frac{27}{4} \frac{d^{7}}{z^{7}}, \quad|z|>d .
\end{array}\right.
\end{aligned}
$$

To express $\phi_{\text {eff }}$ in the form of Eq. (16), we insert Eqs. (A4), (A10), (A12), (A13), (A16), (A18), (A20), and (A22) into Eq. (A2). With some arrangement and expressing the outcome as an expansion of $P_{i} P_{k}^{\prime}$ terms, we obtain Eq. (16) in which coefficients $\phi_{i k}$ are given by

$$
\begin{gathered}
\phi_{00}=A-(\beta / 2)\left(C+\frac{16}{9} D+\frac{16}{3} E+\chi_{d q, d q}^{00}+\chi_{q q, q q}^{00}\right), \\
\phi_{11}=B-(\beta / 2)\left(\chi_{d q, d q}^{11}+H+2 \chi_{d d, q q}^{11}\right), \\
\phi_{22}=\varphi_{q q}-(\beta / 2)\left(\chi_{d q, d q}^{22}+\frac{4}{9} D+8 E\right. \\
\left.-\frac{8}{3} F+G+2 \chi_{00, q q}^{22}+\chi_{q q, q q}^{22}\right), \\
\phi_{33}=-(\beta / 2)\left(\chi_{d q, d q}^{33}+2 \chi_{d d, q q}^{33}\right), \\
\phi_{44}=-(\beta / 2) \chi_{q q, q q}^{44}, \\
\phi_{01}=-\beta\left(\chi_{d d, d q}^{01}+\chi_{d q, q q}^{01}\right), \\
\phi_{02}=-(\beta / 2)\left(\frac{8}{9} D-\frac{8}{3} E+\frac{1}{3} F+\chi_{d q, d q}^{02}+\chi_{q q, q q}^{02}\right), \\
\phi_{03}=-\beta\left(\chi_{d d, d q}^{03}+\chi_{d q, q q}^{03}\right), \\
\phi_{04}=-(\beta / 2)\left(\chi_{d q, d q}^{04}+\chi_{q q, q q}^{04}\right), \\
\phi_{12}=\chi_{d q}^{12}-\beta\left(\chi_{00, d q}^{12}+\chi_{d d, d q}^{12}+\chi_{d q, q q}^{12}\right),
\end{gathered}
$$

$$
\begin{gathered}
\phi_{13}=-(\beta / 2)\left(\chi_{d q, d q}^{13}+2 \chi_{d d, q q}^{13}\right), \\
\phi_{14}=-\beta \chi_{d q, q q}^{14}, \\
\phi_{23}=-\beta\left(\chi_{d d, d q}^{23}+\chi_{d q, q q}^{23}\right), \\
\phi_{24}=-(\beta / 2)\left(\chi_{d q, d q}^{24}+\chi_{q q, q q}^{24}\right), \\
\phi_{34}=-\beta \chi_{d q, q q}^{34} .
\end{gathered}
$$

Here, the $\phi_{i k}(z)$ coefficients are those with $i \leqslant k$. We can introduce the $\phi_{i k}(z)$ coefficient for $k>i$, such that $\phi_{i k}(z)$ $=\phi_{k i}(z)$. Note that all the coefficients satisfy the condition $\phi_{i k}(z)=(-1)^{(i+k)} \phi_{i k}(-z)$.

\section{APPENDIX B}

In this section, we present a list of the calculated integral $\Phi_{i k}=\int_{-\infty}^{+\infty} \phi_{i k}(z) d z$ which is required to deduce Eqs. (27) and (29):

$$
\begin{gathered}
\Phi_{01}=\Phi_{02}=\Phi_{03}=\Phi_{04}=\Phi_{12}=\Phi_{14}=\Phi_{23}=\Phi_{24}=\Phi_{34}=0 \\
\Phi_{00}=-\frac{16}{3} \pi \epsilon d^{3}\left\{1+\frac{\epsilon}{k_{B} T}\left(\frac{2}{3}+\frac{1}{12} \frac{\mu_{0}^{4}}{\epsilon^{2} d^{6}}\right.\right. \\
\left.\left.+\frac{3}{20} \frac{\mu_{0}^{2} Q^{2}}{\epsilon^{2} d^{8}}+\frac{3}{20} \frac{Q^{4}}{\epsilon^{2} d^{10}}\right)\right\} \\
\Phi_{11}=\frac{8}{3} \pi \mu_{0}^{2}\left(1-\frac{243}{875} \frac{Q^{2}}{k_{B} T d^{5}}\right) \\
\Phi_{22}=\frac{4}{3} \frac{\pi}{k_{B} T d^{3}}\left(\frac{1}{15} \mu_{0}^{4}-\frac{3}{35} \frac{\mu_{0}^{2} Q^{2}}{d^{2}}+\frac{12}{49} \frac{Q^{4}}{d^{4}}\right) \\
\Phi_{33}=-\frac{108}{175} \pi \frac{\mu_{0}^{2} Q^{2}}{k_{B} T d^{5}}, \\
\Phi_{13}=\frac{48}{875} \pi \frac{\mu_{0}^{2} Q^{2}}{k_{B} T d^{5}} .
\end{gathered}
$$

[1] J.M. Haile, C.G. Gray, and K.E. Gubbins, J. Chem. Phys. 64, 2569 (1976).

[2] P.I.C. Teixeira and M.M. Telo da Gama, J. Phys.: Condens. Matter 3, 111 (1991).

[3] P. Frodl and S. Dietrich, Phys. Rev. E 48, 3741 (1993).

[4] P.I.C. Teixeira and M.M. Telo da Gama, J. Phys.: Condens. Matter 14, 12159 (2002).

[5] C.E. Woodward and S. Nordholm, J. Phys. Chem. 92, 501 (1988).
[6] M.J. Stevens and G.S. Grest, Phys. Rev. E 51, 5976 (1995).

[7] B. Groh and S. Dietrich, Phys. Rev. E 53, 2509 (1996).

[8] K. Kiyohara, K.J. Oh, X.C. Zeng, and K. Ohta, Mol. Simul. 23, 95 (1999).

[9] V.B. Warshavsky, T.V. Bykov, and X.C. Zeng, J. Chem. Phys. 114, 504 (2001).

[10] G. Stell, J.C. Rasiah, and H. Narang, Mol. Phys. 23, 393 (1972).

[11] G.N. Patey and J.P. Valleau, J. Chem. Phys. 64, 170 (1976). 
[12] M.S. Ananth, K.E. Gubbins, and C.G. Gray, Mol. Phys. 28, 1005 (1974).

[13] J. Winkelmann, Fluid Phase Equilib. 48, 67 (1989).

[14] G.S. Dubey and S.F. O'Shea, Phys. Rev. E 49, 2175 (1994).

[15] F.H. Stillinger and A. Ben-Naim, J. Chem. Phys. 47, 4431 (1967).

[16] M.A. Wilson, A. Pohorille, and L.R. Pratt, J. Chem. Phys. 90, 5211 (1989).

[17] V.L. Kuz'min, Colloid J. 62, 183 (2000).

[18] A.I. Rusanov and V.L. Kuz'min, Colloid J. USSR 39, 338 (1977).

[19] M.A. Wilson and A. Pohorille, J. Chem. Phys. 95, 6005 (1991).

[20] J. Eggebrecht, K.E. Gubbins, and S.M. Thompson, J. Chem. Phys. 86, 2286 (1987).

[21] S.M. Tompson, K.E. Gubbins, and J.M. Haile, J. Chem. Phys. 75, 1325 (1981).

[22] S. Iatzevich and F. Forstmann, Mol. Phys. 98, 1309 (2000).

[23] P. Frodl and S. Dietrich, Phys. Rev. A 45, 7330 (1992).

[24] P.I.C. Teixeira, B.S. Almeida, M.M. Telo da Gama, J.A. Rueda, and R.G. Rubio, J. Phys. Chem. 96, 8488 (1992).

[25] V. Talanquer and D.W. Oxtoby, J. Chem. Phys. 99, 4670 (1993)

[26] T.V. Bykov and X.C. Zeng, J. Phys. Chem. B 105, 11586 (2001).

[27] V.B. Warshavsky and X.C. Zeng, Phys. Rev. Lett. 89, 246104 (2002)

[28] C.T.R. Wilson, Philos. Trans. R. Soc. London, Ser. A 189, 265 (1897).

[29] H. Rabeony and P. Mirabel, J. Phys. Chem. 91, 1815 (1987).

[30] M. Adachi, K. Okumura, and J.H. Seinfeld, J. Aerosol Sci. 23, 327 (1992).

[31] F. He and P.K. Hopke, J. Chem. Phys. 99, 9972 (1993).

[32] J.L. Katz, J.A. Fisk, and V.M. Chakarov, J. Chem. Phys. 101, 2309 (1994).

[33] D. Kane, G.M. Daly, and M.S. El-Shall, J. Phys. Chem. 99, 7867 (1995).

[34] K.J. Oh, G.T. Gao, and X.C. Zeng, Phys. Rev. Lett. 86, 5080 (2001).
[35] K.C. Russel, J. Chem. Phys. 50, 1809 (1969).

[36] F.M. Kuni, A.K. Shchekin, and A.I. Rusanov, Colloid J. USSR 45, 1093 (1983).

[37] A.I. Rusanov and F.M. Kuni, J. Colloid Interface Sci. 100, 264 (1984).

[38] E.N. Brodskaya and A.I. Rusanov, Colloid J. USSR 45, 636 (1983).

[39] E.N. Brodskaya, A.P. Lyubartsev, and A. Laaksonen, J. Chem. Phys. 116, 7879 (2002).

[40] I. Kusaka, Z.-G. Wang, and J.H. Seinfeld, J. Chem. Phys. 102, 913 (1995).

[41] V. Talanquer and D.W. Oxtoby, J. Chem. Phys. 103, 3686 (1995).

[42] T.M. Reed and K.E. Gubbins, Applied Statistical Mechanics (McGraw-Hill, New York, 1973), p. 117.

[43] J.O. Hirschfelder, C.F. Curtis, and R.B. Bird, Molecular Theory of Gases and Liquids (Wiley, New York, 1954), pp. $549-551$.

[44] C.G. Gray and K.E. Gubbins, The Theory of Molecular Fluids (Claredon, Oxford, 1984).

[45] N.E. Carnahan and K.E. Starling, J. Chem. Phys. 51, 635 (1969).

[46] V.B. Warshavsky and X.C. Zeng, J. Chem. Phys. 117, 3982 (2002).

[47] B. Yang, D.E. Sullivan, B. Tjipto-Margo, and C.G. Gray, Mol. Phys. 76, 709 (1992).

[48] B. Yang, D.E. Sullivan, B. Tjipto-Margo, and C.G. Gray, J. Phys.: Condens. Matter 3, F109 (1991).

[49] F.H. Stillinger and A. Rahman, J. Chem. Phys. 60, 1545 (1974).

[50] W.L. Jorgensen, J. Chandrasekhar, J.D. Madura, R.W. Impay, and M.L. Klein, J. Chem. Phys. 79, 926 (1983).

[51] E.N. Brodskaya and V.V. Zakharov, J. Chem. Phys. 102, 4595 (1995).

[52] V.L. Kuz'min and E.N. Brodskaya, Colloid J. 57, 776 (1995).

[53] In this papers the surface potential is measured from the potential of vapor, i.e., $\Delta \varphi=\varphi_{l}-\varphi_{v}$.

[54] M.E. Rose, Elementary Theory of Angular Momentum (Wiley, New York, 1957). 